\title{
Distal vessel stiffening is an early and pivotal mechanobiological regulator of vascular remodeling and pulmonary hypertension
}

Fei Liu, ${ }^{1}$ Christina Mallarino Haeger, ${ }^{2}$ Paul B. Dieffenbach, ${ }^{2}$ Delphine Sicard, ${ }^{3}$ Izabela Chrobak, ${ }^{4}$ Anna Maria F. Coronata, ${ }^{2}$ Margarita M. Suárez Velandia, ${ }^{2}$ Sally Vitali, ${ }^{5}$ Romain A. Colas, ${ }^{6}$

Paul C. Norris, ${ }^{6}$ Aleksandar Marinković, ${ }^{1}$ Xiaoli Liu, ${ }^{2}$ Jun Ma, ${ }^{2}$ Chase D. Rose, ${ }^{5}$ Seon-Jin Lee,,${ }^{2,7}$ Suzy A.A. Comhair, ${ }^{8}$ Serpil C. Erzurum, ${ }^{8}$ Jacob D. McDonald, ${ }^{4}$ Charles N. Serhan, ${ }^{6}$ Stephen R. Walsh, ${ }^{9}$ Daniel J. Tschumperlin, ${ }^{1,3}$ and Laura E. Fredenburgh ${ }^{2}$

${ }^{1}$ Molecular and Integrative Physiological Sciences, Department of Environmental Health, Harvard School of Public Health, Boston, Massachusetts, USA. ${ }^{2}$ Division of Pulmonary and Critical Care Medicine, Department of Medicine, Brigham and Women's Hospital, Boston, Massachusetts, USA. ${ }^{3}$ Department of Physiology and Biomedical Engineering, Mayo Clinic, Rochester, Minnesota, USA. ${ }^{4}$ Lovelace Respiratory Research Institute, Albuquerque, New Mexico, USA. ${ }^{5}$ Department of Anesthesia, Boston Children's Hospital, Boston, Massachusetts, USA. ${ }^{6}$ Center for Experimental Therapeutics and Reperfusion Injury, Department of Anesthesiology, Perioperative and Pain Medicine, Brigham and Women's Hospital, Boston, Massachusetts, USA. ${ }^{7}$ Medical Genomics Research Center, Korea Research Institute of Bioscience and Biotechnology, Daejeon, Korea. ${ }^{8}$ Department of Pathobiology, Lerner Research Institute, Cleveland Clinic, Cleveland, Ohio, USA. 'Division of Infectious Diseases, Brigham and Women's Hospital, Boston, Massachusetts, USA.

Pulmonary arterial (PA) stiffness is associated with increased mortality in patients with pulmonary hypertension (PH); however, the role of PA stiffening in the pathogenesis of $\mathrm{PH}$ remains elusive. Here, we show that distal vascular matrix stiffening is an early mechanobiological regulator of experimental PH. We identify cyclooxygenase-2 (COX-2) suppression and corresponding reduction in prostaglandin production as pivotal regulators of stiffness-dependent vascular cell activation. Atomic force microscopy microindentation demonstrated early PA stiffening in experimental PH and human lung tissue. Pulmonary artery smooth muscle cells (PASMC) grown on substrates with the stiffness of remodeled PAs showed increased proliferation, decreased apoptosis, exaggerated contraction, enhanced matrix deposition, and reduced COX-2-derived prostanoid production compared with cells grown on substrates approximating normal PA stiffness. Treatment with a prostaglandin $\mathrm{I}_{2}$ analog abrogated monocrotaline-induced PA stiffening and attenuated stiffnessdependent increases in proliferation, matrix deposition, and contraction in PASMC. Our results suggest a pivotal role for early PA stiffening in PH and demonstrate the therapeutic potential of interrupting mechanobiological feedback amplification of vascular remodeling in experimental $\mathrm{PH}$.

Authorship note: F. Liu and C.M. Haeger contributed equally to this work.

Conflict of interest: The authors have declared that no conflict of interest exists.

Submitted: February 8, 2016 Accepted: April 26, 2016 Published: June 2, 2016

Reference information: JCI Insight. 2016;1(8):e86987. doi:10.1172/jici.insight.86987.

\section{Introduction}

Pulmonary hypertension $(\mathrm{PH})$ is characterized by pulmonary vascular remodeling, increased pulmonary vascular resistance (PVR), progressive pulmonary arterial (PA) stiffening, and ultimately right ventricular (RV) failure and death (1). In the systemic circulation, arterial stiffness has long been associated with incident hypertension (2) and increased mortality in patients with hypertension (3) and end-stage renal disease (4). Recent studies demonstrate that PA stiffness correlates with mortality in patients with $\mathrm{PH}(5-7)$ and that PA stiffness contributes to RV afterload independent of PVR (8-10). Moreover, measurements of PA stiffness may be more accurate in assessing RV afterload and may be superior to PVR in predicting mortality $(6,8,11)$. However, traditional measures of PA stiffness are largely restricted to large arteries and advanced stages of disease, with little information available about the temporal and spatial changes in vascular stiffness that accompany PH onset and progression. Furthermore, recent work suggests that matrix stiffness itself may amplify and propagate pathologic remodeling (12-14); however, whether PA stiffening 
itself plays a role in the pathogenesis of $\mathrm{PH}$ remains uncertain.

Increases in proximal PA stiffness have been demonstrated in animal models of PH (15-22) and correlate with increases in collagen $(15,16,19)$, elastin $(16,22)$, and collagen cross-linking $(23)$ in large vessels in response to hypoxia. Despite advances in our understanding of large vessel stiffening (24), much less is known about the micromechanical environment in $\mathrm{PH}$ and how the stiffness of the local cellular environment may regulate fundamental aspects of vascular biology. Alterations in tissue stiffness have long been regarded as sequelae of disease; however, emerging studies suggest that the mechanical properties of the matrix may alter cellular activation and promote pathologic tissue remodeling $(13,14,25)$. Changes in the matrix mechanical environment have been shown to dramatically influence cellular morphology, cytoskeletal organization, expression of adhesion molecules, migration, proliferation, and differentiation $(26,27)$ in a number of cell types, including epithelial cells (28), fibroblasts $(13,14,28)$, stem cells (29-32), tumor cells $(33,34)$, and smooth muscle cells $(35-37)$. We have developed methodology to characterize the local elastic properties of the lung using atomic force microscopy (AFM) microindentation $(13,14,38)$. Although AFM is invasive and requires unfixed tissue (38), it allows for unparalleled spatial resolution to measure local vascular tissue stiffening that develops during $\mathrm{PH}$.

We previously demonstrated that cyclooxygenase-2 (COX-2) plays a protective role during hypoxia-induced PH $(1,39)$. Deficiency of COX-2 led to severe PH following hypoxia and was associated with increased contractility and upregulation of the endothelin-1 (ET-1) receptor $\mathrm{ET}_{\mathrm{A}} \mathrm{R}$ in pulmonary artery smooth muscle cells (PASMC) (39). In addition, we recently showed that stiffness-dependent attenuation of COX-2-derived prostaglandin $\mathrm{E}_{2}\left(\mathrm{PGE}_{2}\right)$ synthesis plays a critical role in fibroblast activation in response to matrix stiffening (14). Based on these findings, we hypothesized that matrix stiffness-dependent regulation of COX-2-derived prostanoid expression promotes vascular cell mechanoactivation and drives feedback amplification of vascular remodeling in $\mathrm{PH}$.

To elucidate the effects of pathologic matrix stiffening in $\mathrm{PH}$ pathogenesis, we therefore examined the temporal and spatial distribution of PA stiffening in 2 animal models of $\mathrm{PH}$. We subsequently investigated key remodeling behaviors in human PASMC and pulmonary artery endothelial cells (PAEC) grown on polyacrylamide substrates spanning the stiffness range of normal and remodeled PAs. Additionally, for the first time to our knowledge, we mechanically characterized the stiffness of PAs at the micron scale in human pulmonary arterial hypertension (PAH). Our results demonstrate that PA stiffening originates in the distal vasculature early in the course of experimental $\mathrm{PH}$, significantly before alterations in hemodynamics or RV dysfunction. Increases in PA stiffness bias cellular behavior toward a pathologic remodeling phenotype with enhanced proliferation, attenuated apoptosis, exaggerated traction forces, and increased matrix synthesis.

Mechanistically, we identify decreased COX-2 and corresponding suppression of prostaglandin production as pivotal regulators of stiffness-dependent vascular cell activation, enhanced matrix deposition, and contraction. Our results are consistent with a pivotal role for early matrix stiffening and mechanobiological feedback amplification of vascular remodeling in experimental PH. Early intervention with a long-acting prostacyclin analog to counteract this feedback loop prevented distal vascular stiffening and subsequent vascular and RV sequelae in experimental $\mathrm{PH}$, demonstrating the therapeutic potential of interrupting this adverse mechanobiological feedback loop.

Previous work has demonstrated reduced prostaglandin $\mathrm{I}_{2}\left(\mathrm{PGI}_{2}\right)$ and $\mathrm{PGI}_{2}$ receptor levels in patients with idiopathic and secondary forms of $\mathrm{PH}(40-42)$, although the mechanism underlying this prostaglandin insufficiency has never been elucidated. Our findings now offer mechanistic insight into the underlying loss of endogenous prostaglandins in $\mathrm{PH}$ and suggest that early inhibition of vascular stiffening has the potential to prevent mechanoactivation of vascular cells, restore endogenous prostaglandin levels, and halt progressive vascular remodeling in $\mathrm{PH}$.

\section{Results}

Increased PA stiffness at the micron scale in rat PH models and human PAH. To mechanically characterize the intrapulmonary arterial vasculature and the extent of stiffness changes in experimental $\mathrm{PH}$, we used AFM to examine PA elasticity in the monocrotaline (MCT) and SU5416/hypoxia rat models at the time point of established disease. While AFM is an invasive technique with inherent limitations (see Discussion), it provided the necessary spatial resolution to measure mechanical changes in the small arterioles that undergo remodeling in these disease models. Four weeks following MCT, rats exhibited significant increases in 

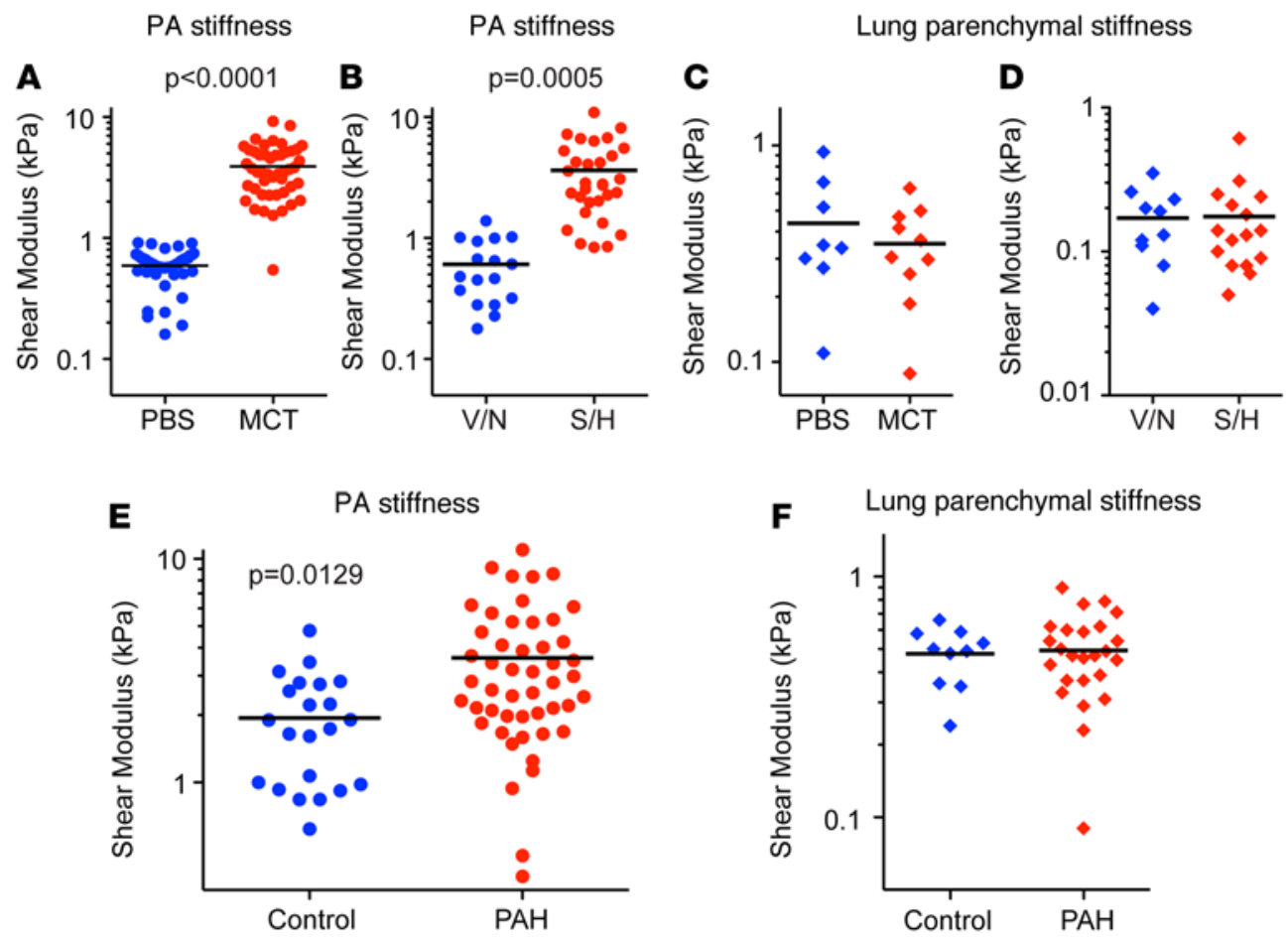

Figure 1. Increased PA stiffness in rat pulmonary hypertension models and human PAH. (A and C) Sprague-Dawley rats were treated with monocrotaline (MCT) or PBS ( $n=6$ per group) and lungs harvested after 4 weeks. (B and D) Sprague-Dawley rats were treated with SU5416 (S) or vehicle (V), exposed to hypoxia (H) or normoxia (N) for 3 weeks, and then returned to normoxia for an additional 5 weeks ( $n=4$ per group). Pulmonary arterioles (PA) $<100 \mu \mathrm{m}$ ( $\mathbf{A}$ and B) and lung parenchyma (C and D) were mechanically characterized via AFM microindentation. Horizontal lines represent the mean shear modulus of each group, and each symbol corresponds to 1 PA (A and B) or parenchymal region (C and $\mathbf{D})$. Statistical significance was determined by a mixed model with treatment as a fixed effect and individual rats as a random effect. (E and $\mathbf{F}$ ) AFM microindentation was used to mechanically characterize $P A s$ (E) and lung parenchyma (F) in human lung samples from PAH $(n=17$; IPAH $n=8, \mathrm{FPAH} n=3$, APAH $n=6)$ and control subjects $(n=7)$. Horizontal lines represent the mean shear modulus of each group, and each symbol corresponds to 1 PA (E) or parenchymal region (F). Statistical significance was determined by a mixed model with disease state as a fixed effect and subgroups and individual patients as nested fixed effects.

PA shear modulus (3.9 kPa) compared with PBS-treated controls $(0.6 \mathrm{kPa})$ (Figure 1A and Supplemental Figure 1A; supplemental material available online with this article; doi:10.1172/jci.insight.86987DS1). To confirm these findings in a model that more closely mimics human PAH, we subjected rats to the SU5416/ hypoxia model $(43,44)$, which is characterized by significant increases in RV systolic pressure (RVSP), RV hypertrophy (RVH), and exaggerated vascular remodeling (Supplemental Figure 2). Similar to the MCT model, PAs from vehicle/normoxia-exposed animals were highly compliant $(0.6 \mathrm{kPa})$, but 8 weeks after SU5416/hypoxia, PAs $<100 \mu \mathrm{m}$ demonstrated substantial increases in shear modulus (3.6 kPa) (Figure 1B and Supplemental Figure 1B). The stiffness of the lung parenchyma was not increased in MCT-treated or SU5416/hypoxia-exposed rats compared with controls (Figure 1, C and D, and Supplemental Figure 1, C and D), demonstrating a localized vascular stiffening response in these PH models.

To ascertain the relevance of these intrapulmonary vascular stiffness changes within the context of human PAH, we used the same technique to measure PA stiffness in explanted lungs obtained from patients with Group 1 PAH who underwent lung transplantation compared with lungs from organ donors whose lungs were deemed not suitable for transplantation (Supplemental Tables 1 and 2). Due to limitations of tissue availability and sample volume, we were able to identify and analyze 21 vessels from 8 patients with idiopathic PAH (IPAH), 10 vessels from 3 patients with familial PAH (FPAH), and 18 vessels from 6 patients with associated PAH (APAH) compared with 22 vessels from 7 unaffected donors. Stiffness values were more broadly varied in the human disease and control tissues than in rats studied using the standardized disease models, but despite the considerable heterogeneity within and across individual vessels, the mean shear modulus of PAs from PAH patients $(3.6 \mathrm{kPa})$ was significantly increased compared with PAs 
$\mathbf{A}$
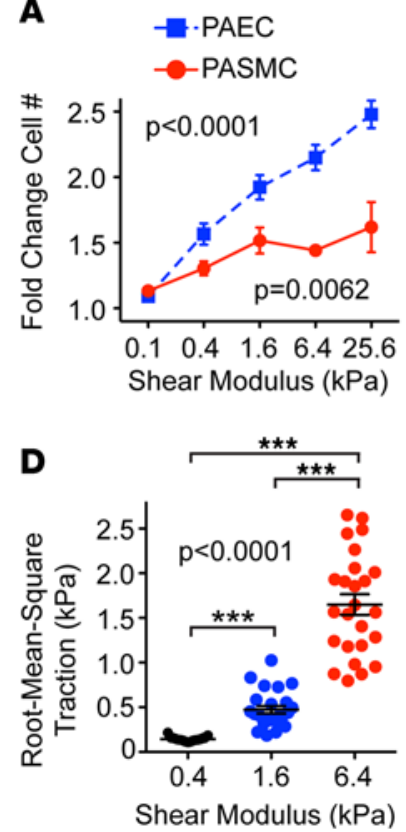

B

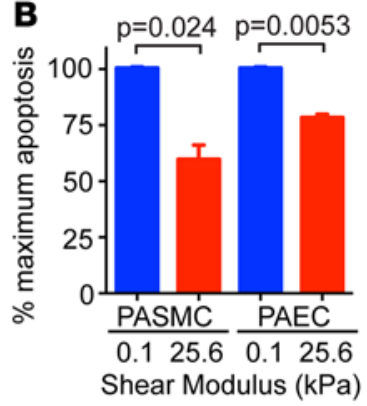

E

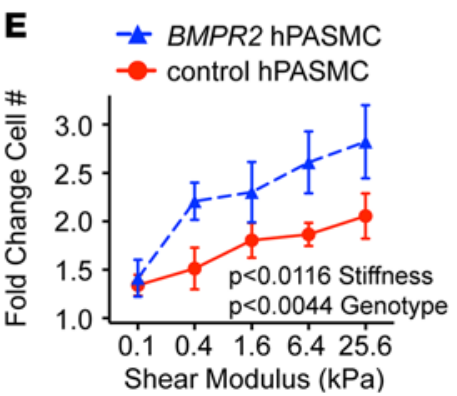

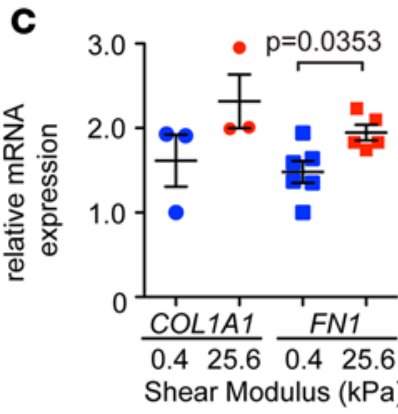

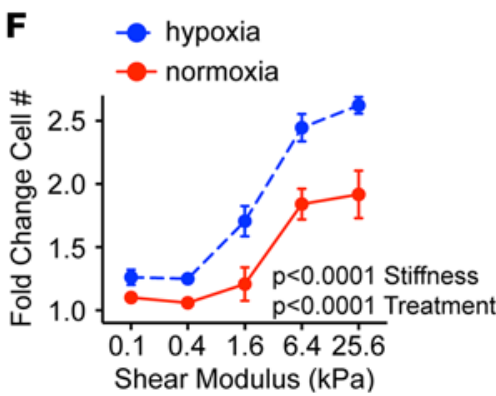

Figure 2. Increased matrix stiffness leads to remodeling responses in PASMC and PAEC. Human PASMC and PAEC were plated on polyacrylamide substrates with stiffness of 0.1-25.6 kPa. After 48 hours, (A) cell density was determined and fold change expressed as the ratio of cell number at 48 hours vs. 4 hours ( $n=7-10$; statistical significance determined by the Kruskal-Wallis 1-way ANOVA). (B) Apoptosis was quantified and percent apoptosis normalized to maximum apoptosis at $0.1 \mathrm{kPa}$ ( $n=2$ independent experiments performed in triplicate; statistical significance determined by the Mann-Whitney $U$ test). (C) qPCR was performed for COL1A1 and FN1 in PASMC and normalized to GAPDH expression ( $n=3-6$ per stiffness; statistical significance determined by the Mann-Whitney $U$ test). (D) Traction forces were quantified in PASMC by measuring fluorescent bead position displacement and calculating root-mean-square traction ( $n=25$ cells per stiffness; $P<0.0001$ by the Kruskal-Wallis 1-way ANOVA, ${ }^{* *} P<0.001$ by Dunn's post test). (E) BMPR2 and control PASMC were plated on gels with stiffness of $0.1-25.6 \mathrm{kPa}(n=2$ independent experiments performed in triplicate; statistical significance determined by 2-way ANOVA, $P<0.0116$ stiffness, $P<0.0044$ genotype, $P=0.5911$ interaction. (F) PASMC were plated on discrete stiffness gels and exposed to normoxia or hypoxia. Cell density was determined at 48 hours and normalized to 4 hours ( $n=3$ independent experiments performed in triplicate; statistical significance determined by 2 -way ANOVA, $P<0.0001$ stiffness, $P<$ 0.0001 treatment, $P=0.0358$ interaction). Data represent the mean and SEM.

in control lung tissue (Figure 1E and Supplemental Figure 1E). Analysis of the lung parenchyma demonstrated no difference in stiffness between PAH patients and controls, again consistent with focal changes localized to the pulmonary vasculature in human PAH (Figure $1 \mathrm{~F}$ and Supplemental Figure 1F). These first measurements of PA stiffness at the micron scale in human and experimental $\mathrm{PH}$ provide a pathophysiological range $(\sim 0.1-20 \mathrm{kPa})$ over which to study vascular cellular function in vitro in $\mathrm{PH}$.

Increased matrix stiffness leads to remodeling responses in PASMC and PAEC. To elucidate whether increases in matrix stiffness evoke vascular remodeling responses in PASMC and PAEC, we generated polyacrylamide gels with discrete shear moduli $(14,45)$ that span the range of PA stiffness observed in human and experimental $\mathrm{PH}$. Increases in substrate stiffness led to significant and proportional increases in proliferation in both PASMC and PAEC (Figure 2A). Furthermore, PASMC and PAEC demonstrated significant attenuation in apoptosis on a stiff matrix (Figure 2B). To investigate whether increases in stiffness alter matrix synthesis, we analyzed type I collagen and fibronectin expression. PASMC grown on a stiff substrate demonstrated a significant increase in mRNA expression of FN1 and a trend toward increased expression of COL1A1 (Figure 2C) compared with PASMC grown on a soft substrate. To determine whether stiffening of the mechanical environment alters PASMC contractility, we used traction force microscopy $(39,46,47)$. PASMC exerted weak traction forces when cultured on soft matrices with a shear modulus approximating normal PAs but demonstrated a dramatic increase in contractile force generation when grown on matrices with a stiffness approximating remodeled PAs (Figure 2D).

To examine whether this stiffness-dependent remodeling phenotype is exaggerated in PAH patients, we cultured bone morphogenetic protein receptor type II-mutant (BMPR2-mutant) PASMC on polyacrylamide gels. Interestingly, BMPR2-mutant PASMC had significantly enhanced proliferation compared with 
A
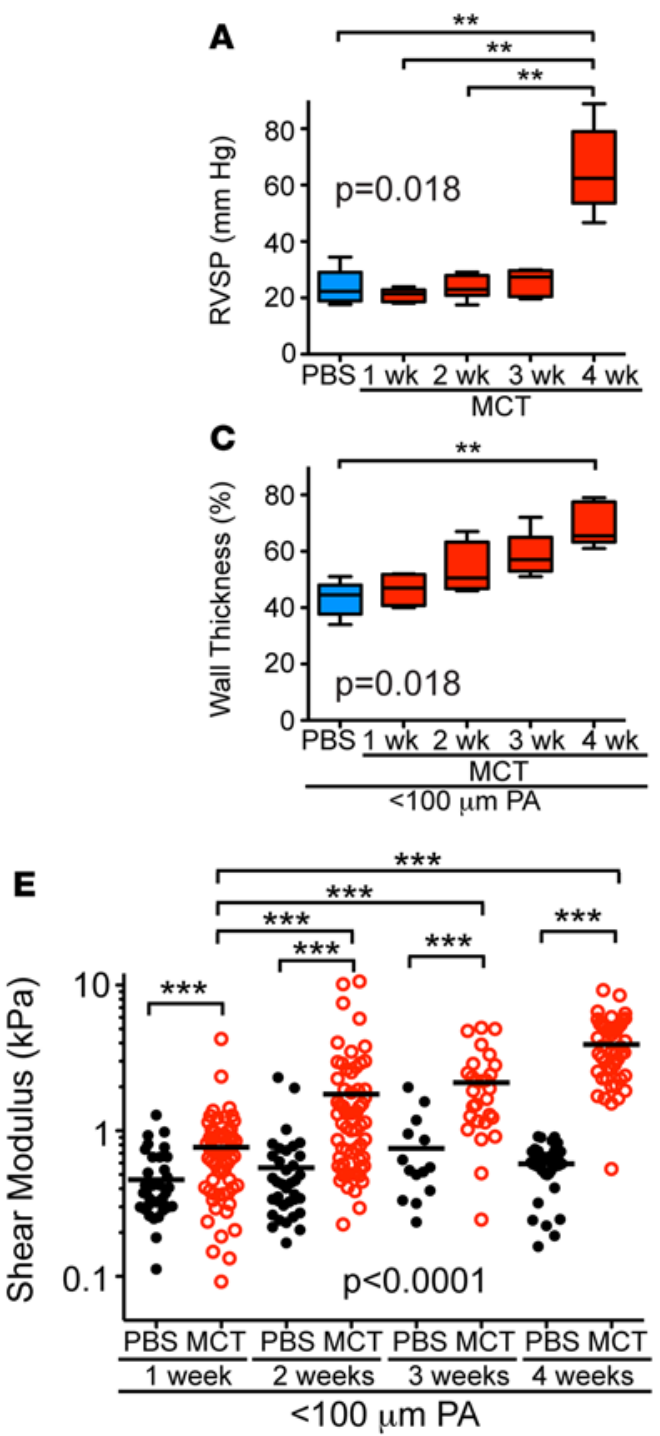
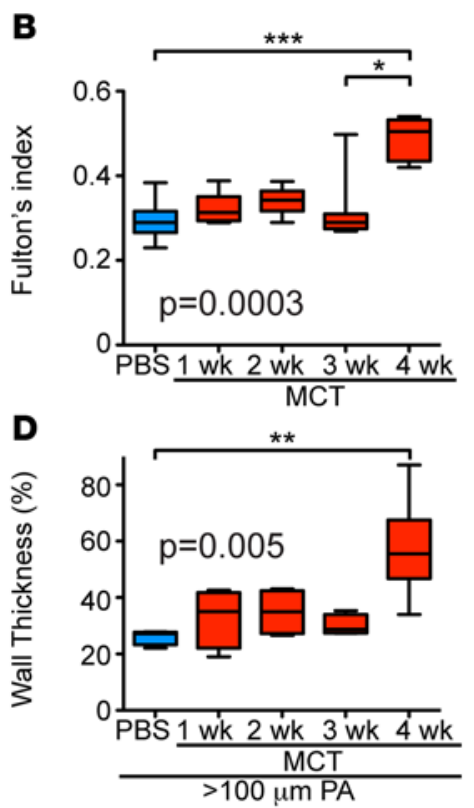

$\mathbf{F}$

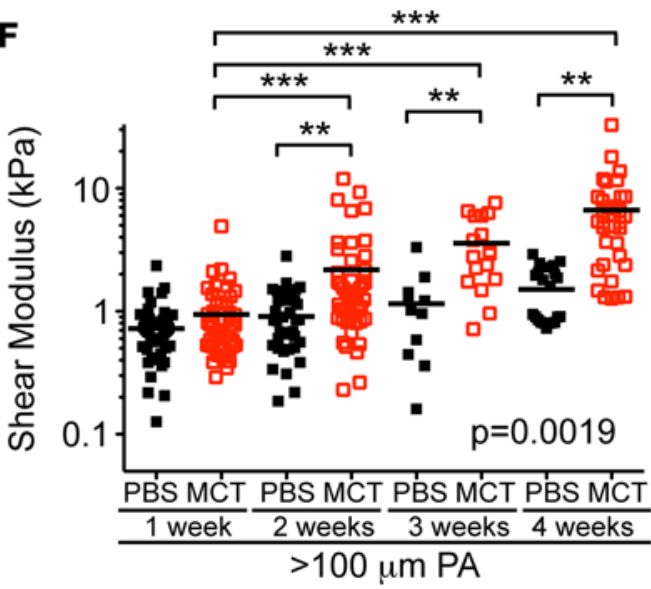

Figure 3. Distal PA stiffening occurs early in MCT-induced pulmonary hypertension. Male Sprague-Dawley rats were treated with monocrotaline (MCT) or PBS ( $n=6-8$ per time point) and harvested at serial time points following MCT. (A) RVSP and (B) Fulton's index were measured weekly for 4 weeks following MCT. Quantification of wall thickness of (C) PAs $<100 \mu \mathrm{m}$ and (D) PAs $>100 \mu \mathrm{m}$. Data represent 25th-75th percentiles (box), median (line), and 5th and 95th percentiles (whiskers). Statistical significance was determined by one-way ANOVA followed by Dunn's post test for multiple comparisons ( $\left.{ }^{*} P<0.05 ;{ }^{* *} P<0.01 ;{ }^{* *} P<0.001\right)$. AFM microindentation was used to mechanically characterize (E) PAs $<100 \mu \mathrm{m}$ and (F) PAs $>100 \mu \mathrm{m}$. Horizontal lines represent the mean shear modulus of each group, and each symbol corresponds to 1 PA. Statistical significance was determined by a mixed model with treatment as a fixed effect and individual rats as a random effect followed by Dunnett's test for multiple comparisons $\left({ }^{*} P<0.05 ;{ }^{*} P<0.01 ;{ }^{* * *} P<0.001\right.$ ).

control PASMC when grown on stiff substrates but similar proliferation as control PASMC when grown on soft matrices (Figure 2E). To assess whether hypoxia alters the stiffness-mediated remodeling phenotype, PASMC grown on polyacrylamide gels were exposed to hypoxia or normoxia. Hypoxia significantly exaggerated stiffness-dependent increases in proliferation in PASMC compared with normoxic cells (Figure 2F). These findings suggest that matrix stiffness may serve as an "additional hit" to propagate cellular activation induced by hypoxia or alteration of BMPR-II signaling in PH.

Distal PA stiffening occurs early in MCT-induced PH. To determine if matrix stiffening occurs early and in a fashion that would allow it to contribute to vascular remodeling and $\mathrm{PH}$ development in vivo, we investigated the temporal and spatial changes in PA stiffness during the evolution of experimental PH. We used AFM microindentation to mechanically characterize PAs $<100 \mu \mathrm{m}$ and $>100 \mu \mathrm{m}$ in rat lung tissue at serial time points following MCT. Animals harvested at 1, 2, and 3 weeks after MCT had no significant hemodynamic 
A

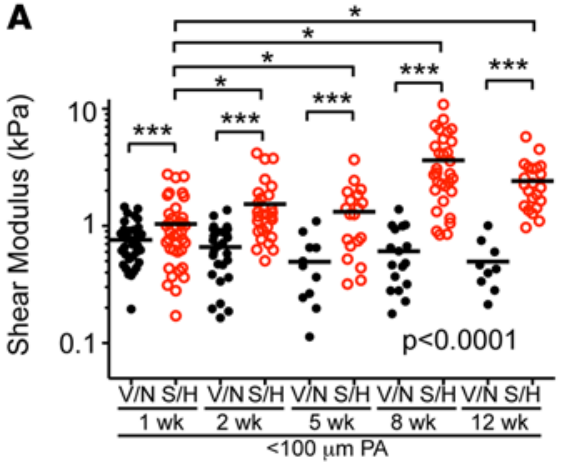

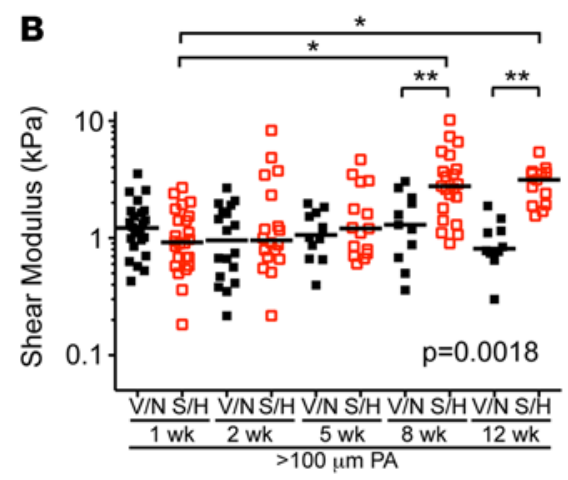
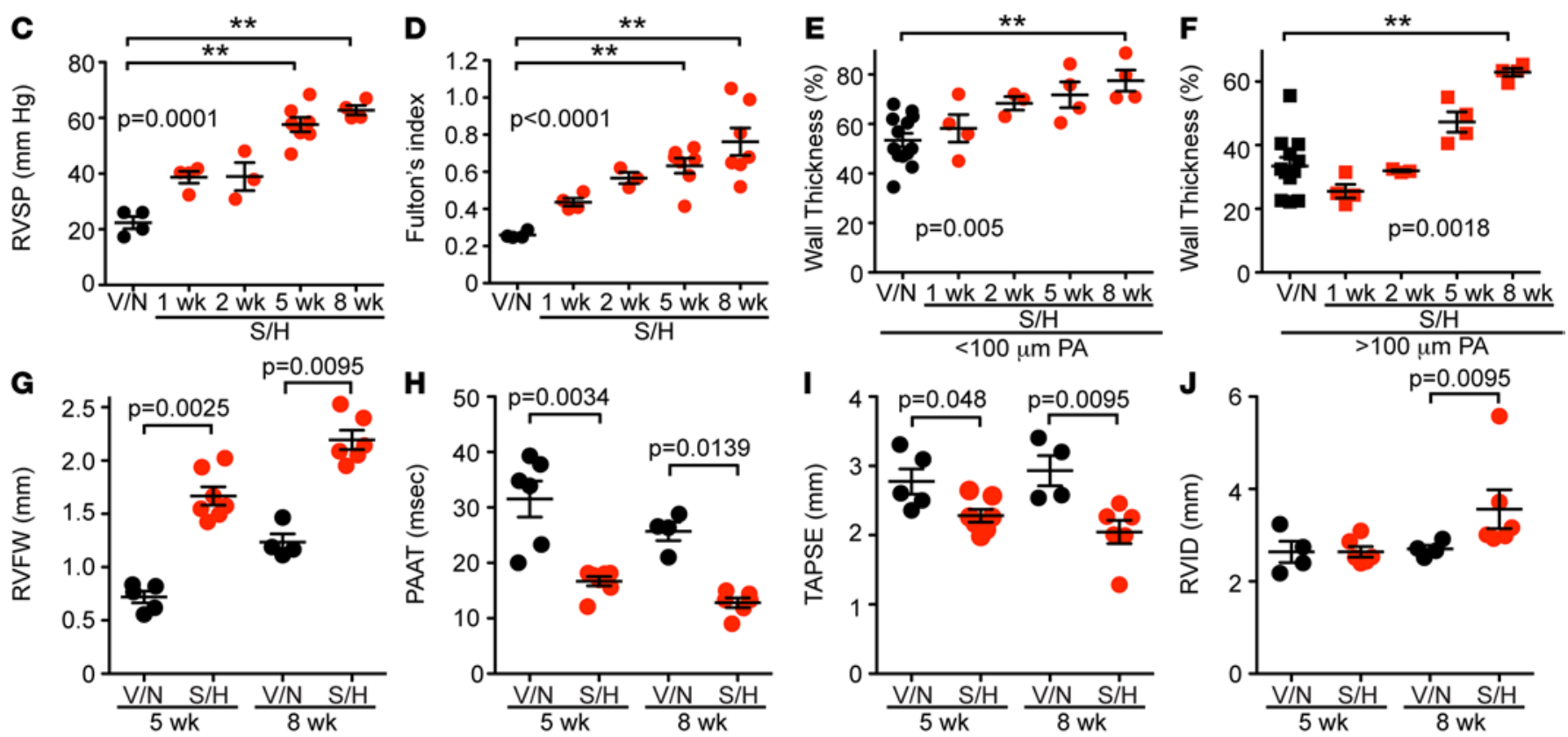

Figure 4. Distal PA stiffening occurs early in the sugen hypoxia PH model. Sprague-Dawley rats were treated with SU5416 (S) or vehicle (V), exposed to hypoxia (H) or normoxia (N) ( $n=4-8$ per time point) for 1, 2, or 3 weeks. Animals exposed to 3 weeks of hypoxia were returned to normoxia for an additional 2, 5, or 9 weeks. PAs $<100 \mu \mathrm{m}$ (A) and PAs $>100 \mu \mathrm{m}$ (B) were mechanically characterized via AFM microindentation. Horizontal lines represent the mean shear modulus of each group, and each symbol corresponds to 1 individual PA. Statistical significance was determined by a mixed model with treatment as a fixed effect and individual rats as a random effect followed by Dunnett's test for multiple comparisons $\left({ }^{*} P<0.05,{ }^{* *} P<0.01,{ }^{* * *} P<\right.$ 0.001). RVSP (C), Fulton's index (D), and PA wall thickness (E and F) were assessed at serial time points. (G-J) Echocardiography was performed ( $n=4-7$ per group) and measurements of RVFW thickness, PAAT, TAPSE, and RVID made at 5 and 8 weeks. Data represent the mean \pm SEM. Statistical significance was determined by the Mann-Whitney $U$ test for pairwise comparisons or by 1-way ANOVA followed by Dunn's post test for multiple comparisons $\left({ }^{*} P<0.05,{ }^{* *} P<0.01,{ }^{* *} P<0.001\right)$.

evidence of PH based on RVSP measurements (Figure 3A). In addition, animals had no significant RVH (Figure 3B) or PA wall thickening (Figure 3, C and D) at these early time points following MCT. Despite no evident PH, MCT-treated animals developed PA stiffening as early as 1 week, with PAs $<100 \mu \mathrm{m}$ demonstrating significant and progressive increases in shear modulus over time (Figure 3E and Supplemental Figure $3 \mathrm{~A}$ ). Increases in stiffness in PAs $>100 \mu \mathrm{m}$ also developed early in the course of disease and preceded hemodynamic and RV changes but followed the PA stiffening seen in smaller, more distal vessels (Figure 3F and Supplemental Figure 3B). Increases in early PA stiffness after MCT correlated with significant increases in mRNA levels of collagen (COL1A1, COL3A1), fibronectin (FN1), and the collagen cross-linking enzyme lysyl oxidase (LOX) (Supplemental Figure 4, A-D). Interestingly, early increases in distal PA stiffening and ECM gene expression all preceded significant increases in RVSP and the development of RVH, all of which were first statistically detectable at 4 weeks following MCT (Figure 3).

Distal PA stiffening occurs early in the SU5416/hypoxia PH model. Similar to the MCT model, PA stiffening occurred early after SU5416/hypoxia and was first observed in small distal vessels (Figure 4A and Supple- 

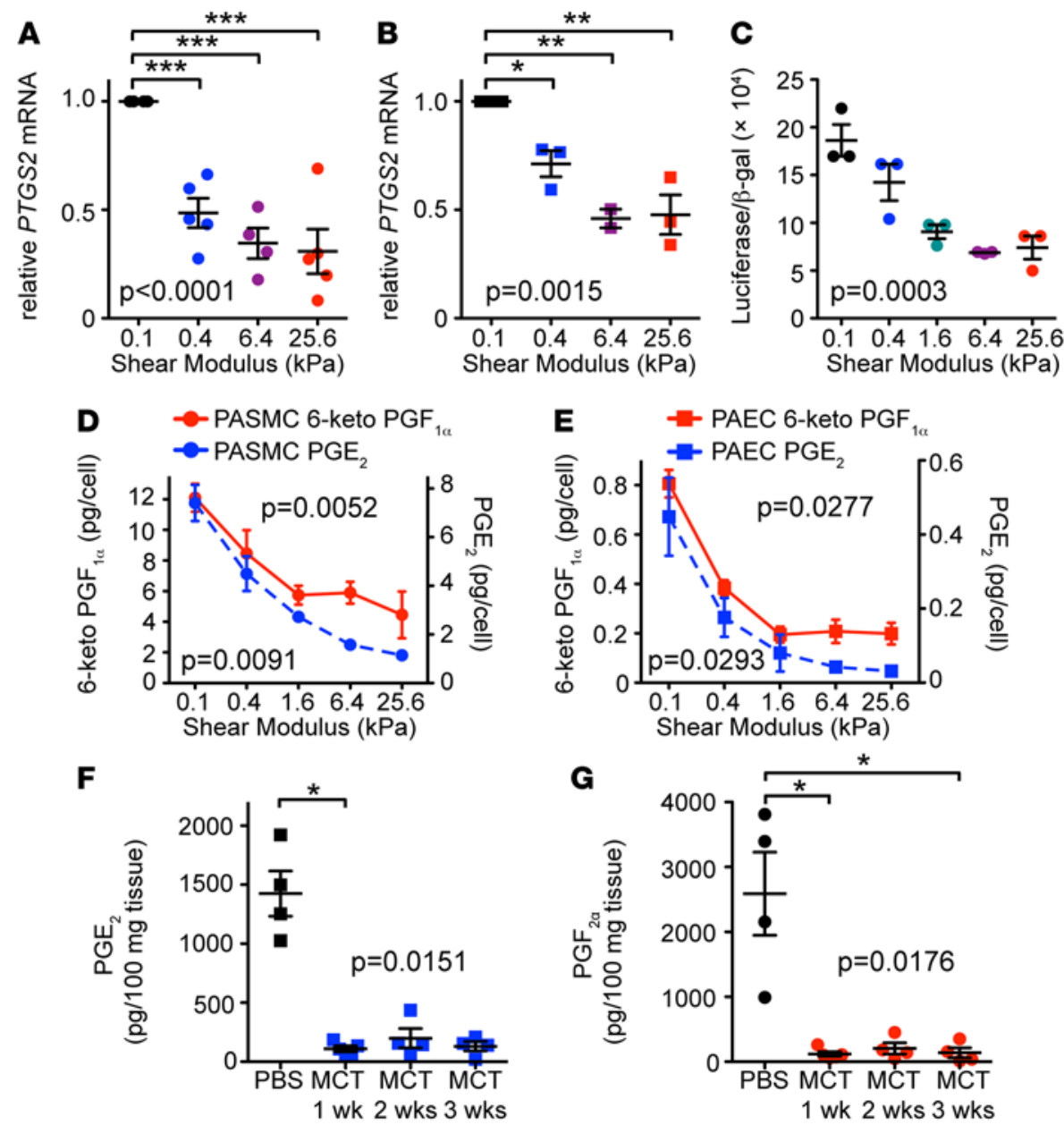

Figure 5. Increased matrix stiffness leads to decreased COX-2 expression and prostanoid production. Human PASMC (A) and PAEC (B) were cultured on polyacrylamide substrates with stiffness of $0.1-25.6 \mathrm{kPa}$. After 48 hours, RNA was isolated and reverse transcribed to CDNA, and qPCR was performed for PTCS2 (COX-2). Results were normalized to GAPDH expression ( $n=2-5$; statistical significance determined by 1-way ANOVA, ${ }^{*} P<0.05$, ${ }^{* *} P$ $<0.01,{ }^{* *} P<0.001$ by Dunnett's post tests). (C) PASMC were transiently transfected with a COX-2 promoter luciferase construct $(-976 /+56)$ via electroporation, and transfected cells were plated on polyacrylamide substrates with stiffness of 0.1-25.6 $\mathrm{kPa}$. After 24 hours, cells were harvested and luciferase and $\beta$-galactosidase assays performed ( $n=3$; statistical significance determined by 1-way ANOVA). ( $\mathbf{D}$ and $\mathbf{E}$ ) After 48 hours, levels of 6-keto PGF $F_{1 \alpha}$ and $P \mathrm{PGE}_{2}$ were measured in media from (D) PASMC and (E) PAEC by ELISA ( $n=3$; statistical significance determined by 1-way ANOVA). (F and $\mathbf{G}$ ) Lipid mediators were extracted from lungs of PBSand MCT-treated rats at 1,2 , and 3 weeks ( $n$ = 4-5 per group) and prostanoids assessed using liquid chromatography-tandem mass spectrometry (LC-MS-MS). Prostanoid levels are expressed as $\mathrm{pg} / 100 \mathrm{mg}$ of lung tissue. Statistical significance was determined by 1-way ANOVA followed by Dunn's post test for multiple comparisons $\left({ }^{*} P<0.05\right.$, ${ }^{* *} P<0.01$, $\left.{ }^{* * *} P<0.001\right)$. Data represent the mean \pm SEM.

mental Figure 3C). SU5416/hypoxia-exposed animals developed PA stiffening as early as 2 weeks in PAs < $100 \mu \mathrm{m}$ with progressive increases in shear modulus over time. After 8 weeks, there were no further increases in shear modulus, although PA stiffening was sustained at 12 weeks (Figure 4A). In vessels $>100 \mu \mathrm{m}$, PA stiffening did not occur until 8 weeks after SU5416/hypoxia, 6 weeks after vascular stiffening had developed in PAs $<100 \mu \mathrm{m}$ (Figure 4B and Supplemental Figure 3D). Similar to the MCT model, early distal PA stiffening was associated with increased expression of ECM encoding genes (Supplemental Figure 4, E-H) and arose prior to significant PA wall thickening (Figure 4, E and F). Interestingly, SU5416/hypoxia-exposed animals showed evidence of $\mathrm{PH}$ at 5 weeks (Figure $4, \mathrm{C}, \mathrm{D}, \mathrm{G}$, and $\mathrm{H}$ ) after the development of distal vessel stiffening at 2 weeks (Figure $4 \mathrm{~A}$ ) but prior to onset of vascular stiffening in more proximal vessels (Figure 4B). In addition, early small vessel stiffening preceded severe RV dysfunction, which developed at 8 weeks (Figure 4, I and J). Taken together, these results suggest that early PA stiffening originates in distal vessels, propagates proximally, and precedes severe hemodynamic changes and RV dysfunction in $\mathrm{PH}$.

Increased matrix stiffness leads to decreased COX-2 expression and prostanoid synthesis. To elucidate the mechanism underlying stiffness-dependent vascular cell activation, we performed a PCR array for a panel of hypertension genes and discovered several genes that were differentially regulated by stiffness. Increased matrix stiffness upregulated several vasoconstrictors (e.g., $A V P$ ) and ion channels (e.g., CLIC-5), while stiffness attenuated genes involved in vasodilation (e.g., PTGS2, encoding COX-2) and calcium transport (e.g., ATP2C1) (Supplemental Table 3). Given our prior work demonstrating enhanced contractility in COX2-deficient PASMC (39), we hypothesized that matrix stiffness-induced COX-2 suppression might drive stiffness-dependent remodeling behaviors. We first confirmed by quantitative PCR (qPCR) that PASMC and PAEC grown on stiff matrices had dramatically reduced expression of PTGS2 (COX-2) compared with cells grown on soft matrices (Figure 5, A and B). Next, to examine whether COX-2 attenuation is controlled at the transcriptional level, we transiently transfected PASMC with a COX-2 promoter reporter 
A

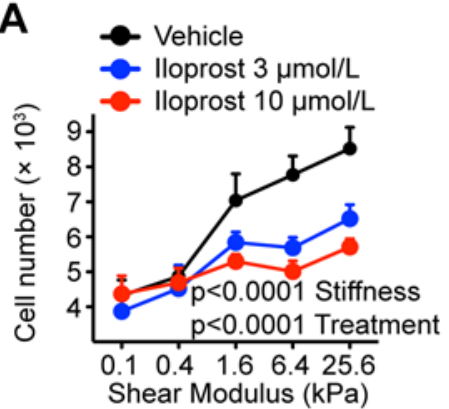

K $\quad p=0.0082$ Stiffness

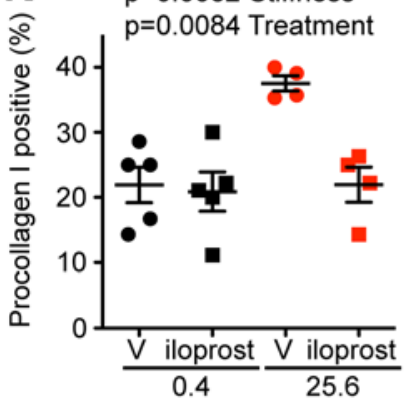

Shear Modulus $(\mathrm{kPa})$

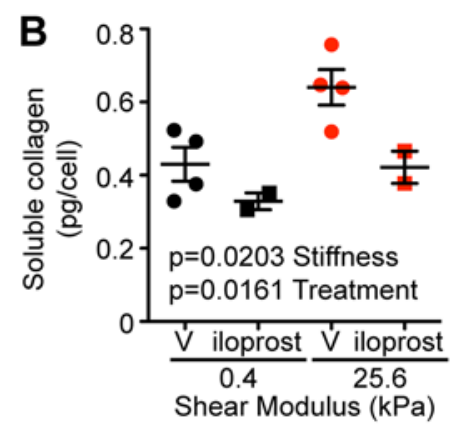

L

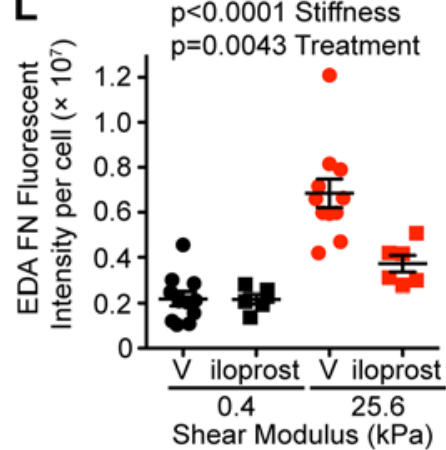

ar Modulus (kPa)
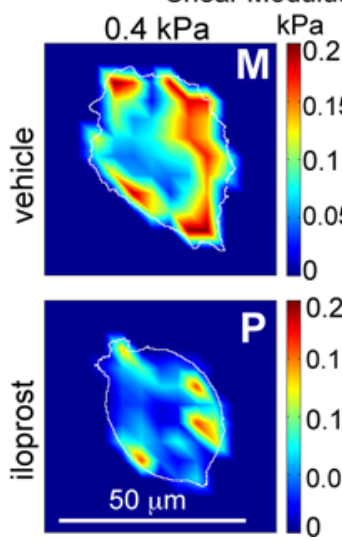

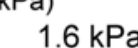

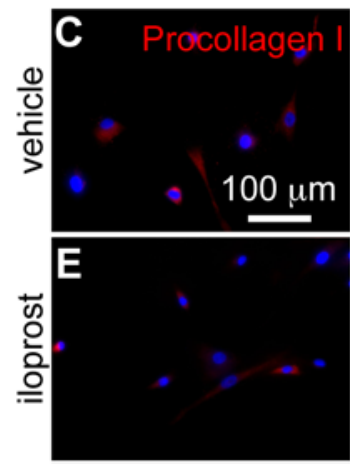
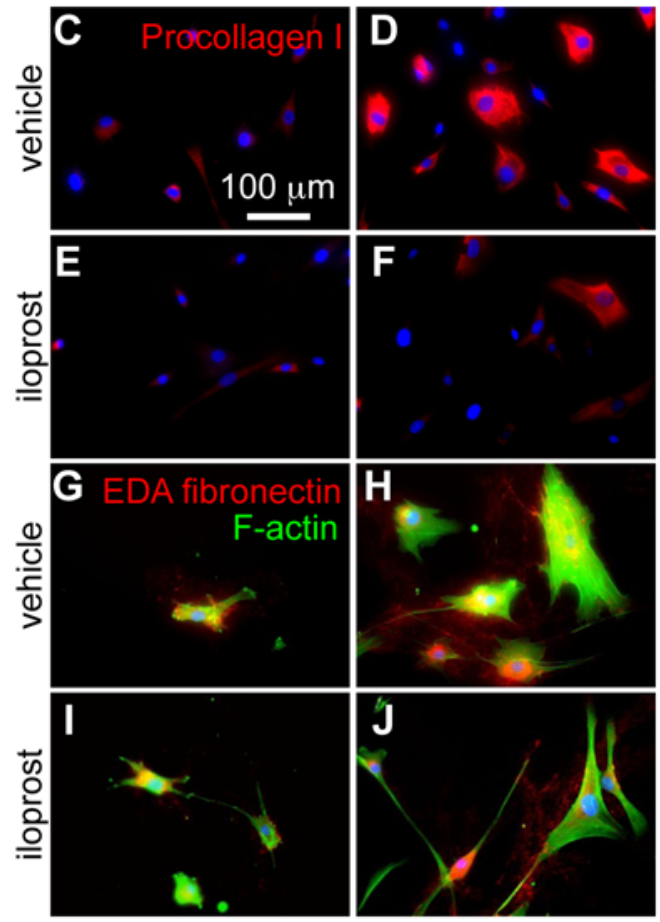

S

$p<0.0001$ Stiffness

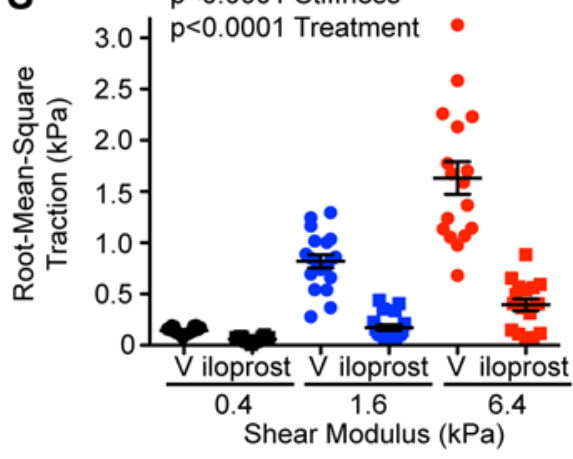

Figure 6. Iloprost attenuates stiffness-dependent remodeling responses and traction forces in PASMC. PASMC were plated on discrete stiffness gels and treated with iloprost ( 3 or $10 \mu \mathrm{mol} / \mathrm{I}$ ) or vehicle for 48 hours. (A) Cell density was determined after 48 hours and normalized to 4 hours ( $n=3-5$; statistical significance determined by 2 -way ANOVA, $P<0.0001$ stiffness, $P<0.0001$ treatment, $P=0.1020$ interaction). (B) Collagen concentration was measured in the media of vehicle- and iloprost-treated PASMC after 48 hours and normalized to cell number ( $n=2-4$; statistical significance determined by 2 -way ANOVA, $P=0.0203$ stiffness, $P=0.0161$ treatment, $P=0.2960$ interaction). (C-J) Representative images of immunofluorescent staining for procollagen I (C-F) and EDA fibronectin (G-J) in vehicle- and iloprost-treated PASMC. Scale bar: $100 \mu$ m. Quantitation of immunostaining for procollagen I (K) $(P=$ 0.0082 stiffness, $P=0.0084$ treatment, $P=0.0184$ interaction by 2 -way ANOVA) and EDA fibronectin (L) $(P<0.0001$ stiffness, $P=0.0043$ treatment, $P$ $=0.0047$ interaction by 2-way ANOVA). (M-R) PASMC were plated on polyacrylamide gels with discrete shear moduli of 0.4, 1.6 , and 6.4 kPa and, after 24 hours, treated with iloprost $(10 \mu \mathrm{mol} / \mathrm{I})$ or vehicle for 30 minutes. Representative traction fields following treatment with vehicle (row 1 , $\mathbf{M - 0 )}$ or iloprost (row 2, P-R). Each column represents discrete substrate stiffness. Color scale indicates magnitude of traction in kPa. Scale bars: 50 um. (S) Quantification of traction forces ( $n=17-20$ cells per group) exerted by vehicle- and iloprost-treated PASMC on discrete stiffness gels $(P<0.0001$ stiffness, $P<0.0001$ treatment, $P<0.0001$ interaction by 2 -way ANOVA). Data represent the mean \pm SEM.

construct that contains key regulatory elements for COX-2 expression. With increasing matrix stiffness, COX-2 promoter activity was significantly downregulated with a $>65 \%$ reduction in COX-2 promoter activity from a soft to stiff mechanical environment (Figure 5C).

We next assessed whether increases in stiffness alter autocrine levels of COX-2-derived prostaglandins. Analysis of key prostanoid mediators in the media demonstrated striking reductions in both 6-keto-PGF ${ }_{1 \alpha}(\mathrm{a}$ $\mathrm{PGI}_{2}$ metabolite) and $\mathrm{PGE}_{2}$ from both PASMC (Figure 5D) and PAEC (Figure 5E) that were inverse to increases in matrix stiffness. We also found that stiffness-dependent attenuation in prostanoid levels in PASMC is COX-2 dependent, as we observed no stiffness-dependent changes in expression of PTGS1 (COX-1) or PTGIR (PGI 
A

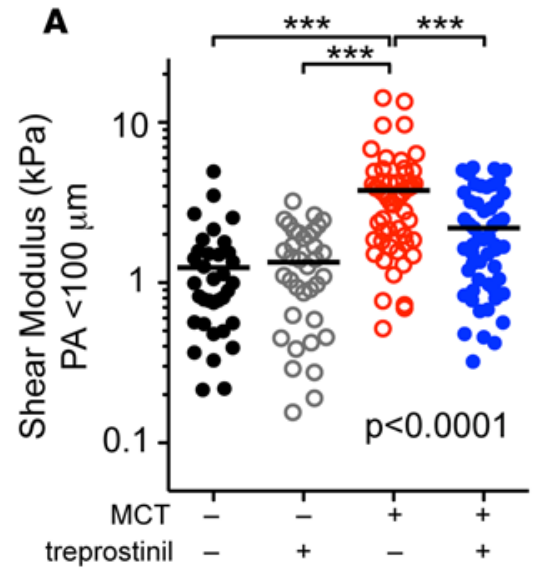

C
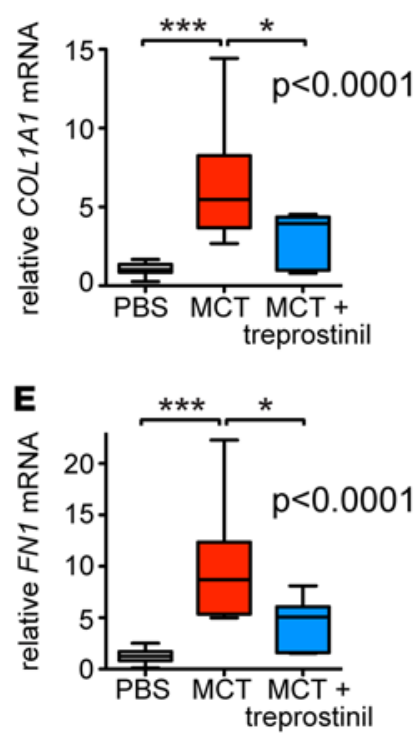

B

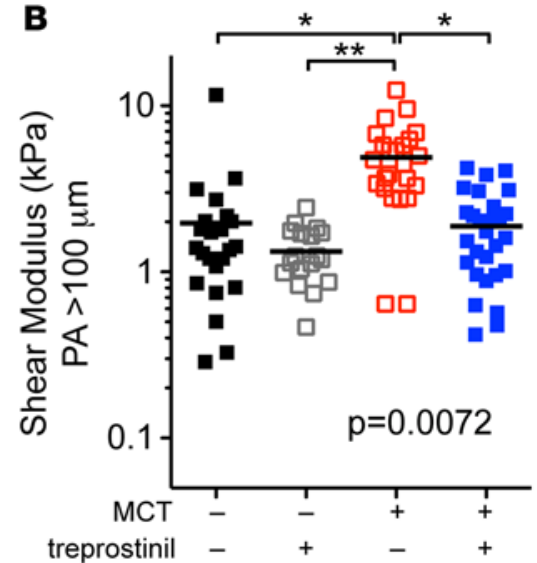

D
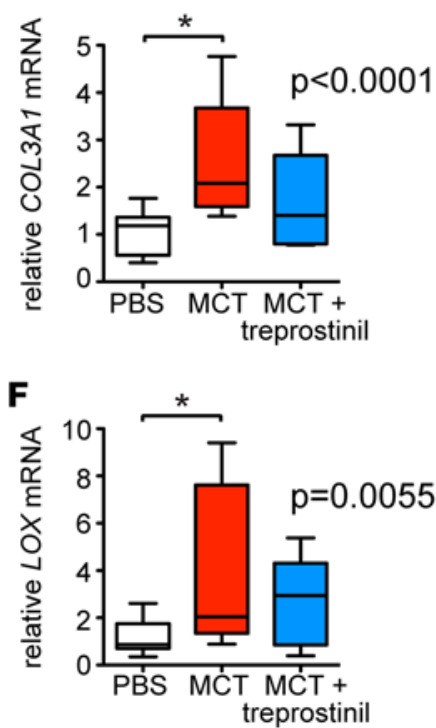

Figure 7. Treprostinil prevents PA stiffening in the MCT model. Sprague-Dawley rats were treated with MCT or vehicle and, after 2 weeks, had s.c. minipumps implanted to deliver i.v. treprostinil (90 $\mathrm{ng} / \mathrm{kg} / \mathrm{min}$ ) or saline. PAs $<100 \mu \mathrm{m}$ (A) and PAs $>100 \mu \mathrm{m}$ (B) were mechanically characterized via AFM microindentation. Horizontal lines represent the mean shear modulus of each group ( $n=4-6$ per group), and each symbol corresponds to 1 individual PA. Statistical significance was determined by a mixed model with treatment as a fixed effect and individual rats as a random effect, followed by Dunnett's test for multiple comparisons $\left({ }^{*} P<0.05\right.$, ${ }^{* *} P$ $\left.<0.01,{ }^{* *} P<0.001\right)$. Lungs were harvested $(n=4-6$ per group) and qPCR performed for (C) COL1A1, (D) COL3A1, (E) FN1, and (F) LOX and normalized to $18 \mathrm{~S}$ ribosomal RNA expression. Data represent 25th-75th percentiles (box), median (line), and 5th and 95th percentiles (whiskers). Statistical significance was determined by 1-way ANOVA followed by Dunn's post test $\left({ }^{*} P<0.05 ;{ }^{* *} P<0.01\right.$; $\left.{ }^{* * *} P<0.001\right)$.

receptor) in PASMC (Supplemental Figure 5). In addition, we analyzed temporal expression of COX-2-derived prostanoids in lung tissue by metabololipidomics in experimental $\mathrm{PH}$. We found that both MCT and SU5416/hypoxia models were characterized by early decreases in prostanoid expression at 1 week, including substantial reductions in $\mathrm{PGE}_{2}, \mathrm{PGD}_{2}$, and $\mathrm{PGF}_{2 a}$, compared with controls (Figure 5, F and G, and Supplemental Figure 6).

Prostacyclin attenuates stiffness-dependent remodeling responses in PASMC and prevents $M C T$-induced PA stiffening. Given these findings, we next investigated whether administration of prostacyclin $\left(\mathrm{PGI}_{2}\right)$ would prevent stiff-

ness-dependent remodeling behaviors in PASMC and inhibit PA stiffening in experimental PH. Treatment of PASMC with the synthetic $\mathrm{PGI}_{2}$ analog iloprost significantly abrogated stiffness-dependent increases in proliferation (Figure $6 \mathrm{~A}$ ) and soluble collagen production (Figure $6 \mathrm{~B}$ ), as well as attenuated procollagen I and fibronectin expression (Figure 6, C-L). Furthermore, iloprost significantly reduced contractile force generation by PASMC grown on both soft and stiff matrices (Figure 6, M-S). To test the efficacy of this strategy in vivo, we also examined whether exogenous treprostinil, a long-acting prostacyclin analog, would inhibit PA stiffening in vivo in experimental PH. Treatment with treprostinil at the time of early MCT-induced PA stiffening, but prior to $\mathrm{PH}$ development, prevented PA stiffening with significant reduction in the shear modulus of both PAs $<100 \mu \mathrm{m}(2.1 \mathrm{kPa})$ and $>100 \mu \mathrm{m}(1.9 \mathrm{kPa})$ compared with saline-MCT controls (PA < $100 \mu \mathrm{m}, 3.8 \mathrm{kPa}$; PA $>100 \mu \mathrm{m}, 4.9 \mathrm{kPa}$ ) (Figure 7, A and B, and Supplemental Figure 7). This reduction in PA stiffness correlated with significant decreases in collagen and fibronectin expression (Figure 7, C-F). Furthermore, inhibition of PA stiffening by treprostinil prevented the development of MCT-induced PH with significant attenuation in RVSP (Figure 8A), RVH (Figure 8B), and PA wall thickening (Figure 8, C-L).

We also investigated whether modulation of COX-2 expression would alter stiffness-dependent cellular behaviors in PASMC. Absence of COX-2 mimicked a stiff matrix environment with enhanced proliferation and matrix protein expression in PASMC, even when grown under physiologic stiffness conditions (Supplemental Figure 8, A and B). In contrast, overexpression of COX-2 mimicked soft matrix conditions with attenuated COL1A1 and FN1 expression, despite a pathologic stiff microenvironment (Supplemental Figure 8, C and D). Taken together, our findings demonstrate that alterations in the mechanical environment play a key role in 


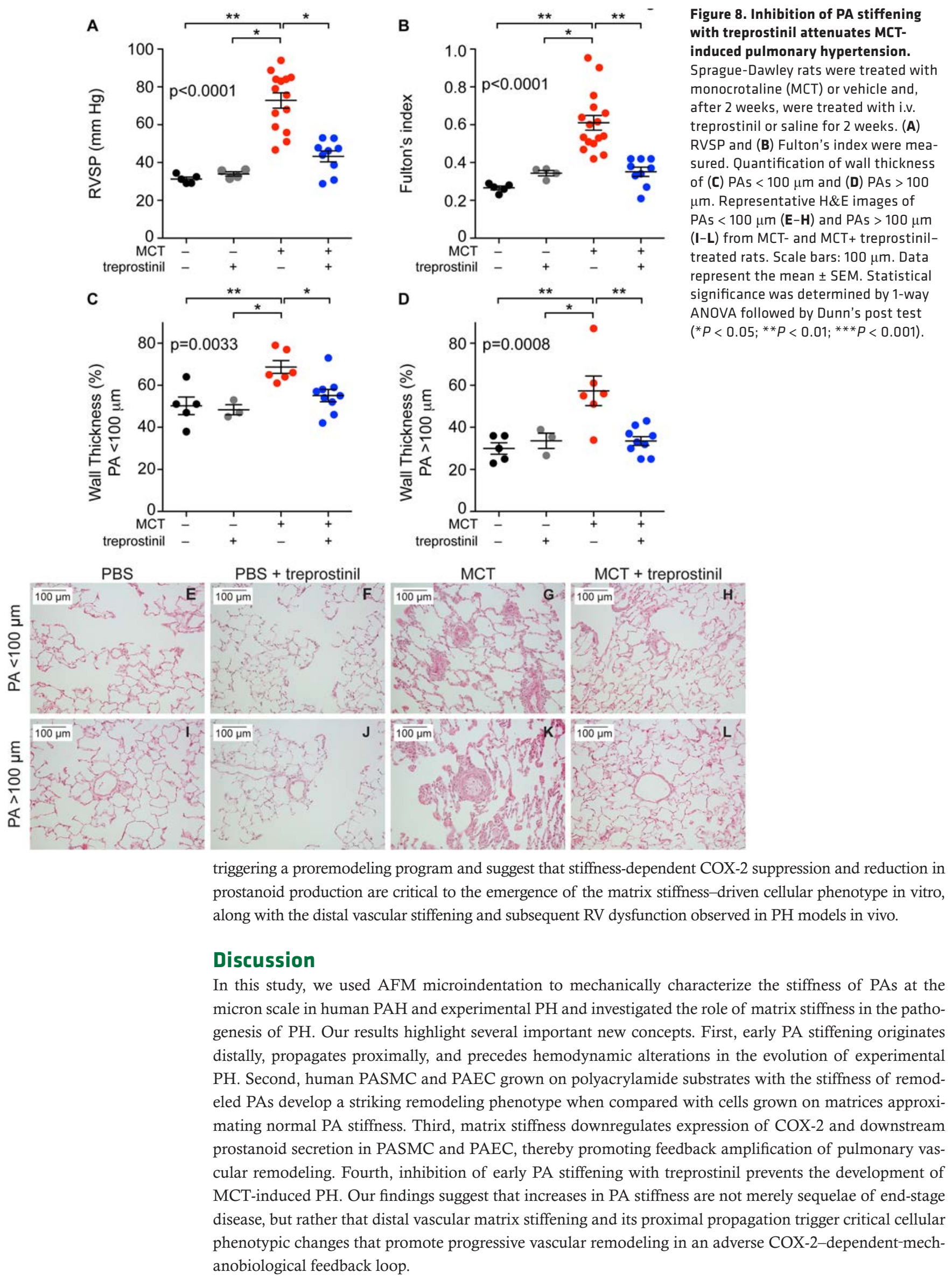


Recent studies demonstrate that PA stiffness is associated with increased mortality in PAH patients $(5-7)$ and may be a better predictor of functional status $(8,11)$ than traditional clinical parameters. Several modalities to estimate PA stiffness in patients have been developed, including right heart catheterization (7), intravascular ultrasound $(6,48)$, cardiac magnetic resonance (CMR) imaging $(5,10,11,49)$, and echocardiography $(8,50,51)$. In animal models, stiffness measurements have focused on isolated vessel mechanical testing (15-17, 19-22), pressure-flow measurements (18), and - more recently — Doppler imaging (21). However, clinical studies $(5,7,8,11,50,51)$ and animal models $(15,16,19,22,52)$ have primarily focused on PA stiffness measured at the macroscopic scale in established disease. In clinical studies, large vessel stiffening measured at the macroscopic scale has been shown to contribute to increased RV workload, as well as to predict clinical outcomes in PAH $(8,9)$. In animal models, large vessel stiffening following exposure to hypoxia has been shown to correlate with increases in vessel wall elastin $(16,22)$ and collagen $(15,16,19)$, as well as increased collagen cross-linking $(23)$.

Although considerable progress has been made in our understanding of large vessel stiffening at the macroscopic level, little is known about small vessel stiffening measured the microscopic level and how micromechanical changes may influence resident vascular cells to promote the development and progression of PH. Using AFM microindentation, our study demonstrates for the first time to our knowledge that PAs develop dramatic mechanical changes at the micron scale in human PAH and experimental models of PH. Compared with controls, patients with Group 1 PAH demonstrated increased PA stiffness, which was similar in magnitude to MCT-treated and SU5416/hypoxia-exposed animals with well-established $\mathrm{PH}$. We also report for the first time to our knowledge that increased PA stiffness occurs early prior to the development of other $\mathrm{PH}$ phenotypes and that inhibition of early PA stiffening prevents the development of PH. Additionally, we found that PA stiffening first arises distally in PAs $<100 \mu \mathrm{m}$, followed by stiffening of more proximal vessels. While measurements of tissue stiffness at the microscopic scale are fundamentally different than traditional measures of PA stiffness at the macroscopic scale and are not sufficient to generate detectable hemodynamic changes, our findings suggest that early changes in the local mechanical environment contribute to progressive vascular remodeling and promote the development of $\mathrm{PH}$. Although recent work suggests that early increases in LOX expression and collagen cross-linking contribute to $\mathrm{PH}$ pathogenesis (23), our findings now provide evidence that vessel wall stiffening is a pivotal early event that propagates pulmonary vascular remodeling and drives progression of $\mathrm{PH}$. These results support recent CMR studies that patients with exercise-induced $\mathrm{PH}$ exhibit changes in PA elasticity before the development of PA pressure elevations at rest (49) and RV dysfunction (10).

The mechanisms underlying PA elasticity changes and how they contribute to $\mathrm{PH}$ pathogenesis are incompletely understood. Prior attention has focused predominantly on flow pulsatility in large vessels and perturbations in shear stress that contribute to remodeling and regulation of vascular tone $(24,53)$. Here, we demonstrate that pathologic matrix stiffening at the micron scale activates PASMC and PAEC to proliferate and triggers PASMC to synthesize matrix and transmit robust contractile forces to the extracellular matrix. This phenotype is exaggerated in BMPR2-mutant PASMC, as well as under conditions of hypoxia, suggesting a synergistic interaction between mechanical forces and hypoxia or altered BMPR-II signaling. Importantly, when BMPR2-mutant PASMC were grown on soft matrices, proliferation was significantly attenuated and similar to control PASMC, suggesting that modulation of matrix stiffness may reverse the proliferative remodeling phenotype. Collectively, our findings suggest that increases in PA stiffness trigger a cellular remodeling program that leads to progressive matrix stiffening and amplification of remodeling in an adverse self-sustaining feedback loop.

We previously demonstrated a central role for the COX-2/ $\mathrm{PGE}_{2}$ pathway in matrix stiffness-driven fibroblast activation (14), and we therefore hypothesized that eicosanoid biosynthesis would be critical to the stiffness-dependent activation of vascular cells. We found that increases in stiffness led to dramatic decreases in levels of the most abundant prostanoids in the pulmonary vasculature, namely 6-keto-PGF $\mathrm{P}_{1 \alpha}$ and $\mathrm{PGE}_{2}$. In addition, we discovered that stiffness suppressed expression of COX-2 in both PAMSC and PAEC, and we demonstrated that stiffness regulates COX-2 expression at the transcriptional level in PASMC. We found that exogenous iloprost can prevent the stiffness-driven shift to a remodeling phenotype in PASMC and can restore cellular behaviors similar to those observed on matrices of physiologic PA stiffness. Importantly, our results also revealed that early administration of treprostinil in vivo at the initiation of PA stiffening, but prior to hemodynamic alterations and development of RVH, completely prevents MCT-induced PH. Future studies will focus on mechanotransduc- 
tion and downstream prostaglandin receptors, given the differential responses of individual prostacyclin analogs on prostanoid receptors (54-57).

Endogenous levels of prostacyclin are reduced in patients with $\mathrm{PAH}(41)$ and exogenous prostanoid therapy remains a cornerstone for the treatment of PAH (58). However, prostacyclin analogs are currently reserved for treatment of severe PAH, typically for those with New York Heart Association (NYHA)/ WHO functional class (FC) III or IV (59). Moreover, recent data from the REVEAL registry suggest that prostanoid therapy is not consistently being prescribed in patients with severe PAH (60), despite current guideline recommendations (59). Our results provide an explanation for the reduced prostacyclin levels in $\mathrm{PAH}$ patients and suggest that early vascular stiffening and suppression of endogenous prostanoid levels underlie the mechanoactivation of vascular cells and disease progression in PAH. Furthermore, our findings have important clinical implications and argue for initiation of prostanoid therapy early in the disease course to interrupt mechanopathobiologic signaling from the matrix to resident vascular cells and arrest progression of vascular remodeling. This supports emerging evidence that PAH patients with less severe disease may benefit from prostanoids $(61,62)$, a treatment strategy that now may be more feasible with the recent FDA approval of oral prostacyclin analogs $(63,64)$.

To gain new insight into micromechanical remodeling in $\mathrm{PH}$, we applied AFM microindentation to measure PA stiffness at the cellular scale within the intact native lung architecture $(38,65)$. Although AFM is invasive, it allowed us to assess the mechanical properties of intrapulmonary vessels with micron-scale spatial resolution. There are clear advantages to our approach, including high spatial resolution, small deformations, and sensitivity to modest changes in mechanical properties. However, AFM is not without limitations. Sample preparation for AFM characterization requires sectioning of unfixed tissue; thus, the boundary conditions for vessels and surrounding parenchyma are modified from their in situ state. Nevertheless, AFM has been used previously to characterize remodeling-associated changes in vascular wall stiffness using similar approaches $(12,66)$. As our AFM measurements were made at room temperature in nonperfused tissue, the measured tissue stiffness does not take into account active cellular contributions, as well as possible contributions from changes in transmural distending pressure (67) or vascular flow. However, as this study focused on distal PA stiffness, we suspect that flow and transmural pressure contributions to stiffness are small and that our measurements capture changes in vascular wall stiffness associated with ECM remodeling (68). Additional limitations of AFM include sampling challenges associated with the small spatial scale of measurements and inherent biological heterogeneity across and within vessels. In human samples, we observed smaller differences in PA stiffness between control and $\mathrm{PAH}$ patients than differences observed in our animal PH models. Potential explanations for this difference in magnitude include lack of OCT inflation in human lungs that may have affected AFM measurements, heterogeneity of disease subtype and severity in PAH patients, and demographics of human subjects. Furthermore, treatment with prostanoids and other medications at the time of lung transplantation may have confounded stiffness assessment in human PAH tissue. In addition, control samples were procured from donors with inadequate organ quality who may have had unknown pulmonary vascular elasticity changes. Despite these challenges, we observed statistically significant alterations in vessel stiffness in both human $\mathrm{PAH}$ and experimental models of $\mathrm{PH}$, emphasizing the robust nature of distal vascular stiffening in $\mathrm{PH}$.

In summary, we demonstrate that under conditions of pathologic PA stiffness, suppression of COX-2 expression and $\mathrm{PGI}_{2}$ synthesis triggers a key shift in cellular behaviors to a remodeling phenotype with enhanced proliferation, matrix deposition, and contractility, leading to further increases in arterial stiffness and amplification of remodeling. Furthermore, our findings suggest that, once increases in PA stiffness are established, propagation of the vascular remodeling process is self-sustaining via activation of PASMC and PAEC and attenuation of COX-2 expression in a mechanobiological feedback loop. Although long regarded as a consequence of vascular remodeling, our results now demonstrate that increases in PA stiffness contribute to the pathogenesis of $\mathrm{PH}$ and suggest that interrupting this stiffness-dependent feedback cycle may offer a novel therapeutic approach for the treatment of patients with $\mathrm{PH}$.

\section{Methods}

\section{Chemicals and reagents}

Iloprost was obtained from Cayman Chemical. All cell culture reagents were obtained from Lonza unless 
otherwise specified. Treprostinil was provided by United Therapeutics. All other reagents were obtained from Sigma-Aldrich unless otherwise specified.

\section{Animals}

PASMC were isolated from mice that were WT $(+/+)$ or homozygous null $(-/-)$ for targeted disruption of COX-2 (B6:129S7-Ptgs2tm1Jed, The Jackson Laboratory). Animals were obtained via heterozygous breeding, and genotypes were determined by a PCR protocol provided by the Jackson Laboratory with modification.

MCT model. Adult male Sprague-Dawley rats weighing approximately 250-280 g (Charles River Laboratories) were injected with a single dose of MCT $(50 \mathrm{mg} / \mathrm{kg}$ ) or vehicle (PBS) s.c. (69). For the treprostinil experiment, 2 weeks following MCT, male Sprague-Dawley rats had a s.c. minipump implanted with a cannula inserted into the left jugular vein to deliver i.v. treprostinil ( $90 \mathrm{ng} / \mathrm{kg} / \mathrm{min})$ or saline for an additional 2 weeks.

Sugen hypoxia model. Adult male Sprague-Dawley rats weighing 200-220 g were injected s.c. with a single dose of SU5416 (Sigma-Aldrich) $(20 \mathrm{mg} / \mathrm{kg}$ ) or vehicle (DMSO) followed by exposure to hypoxia $(10 \%$ $\left.\mathrm{O}_{2}\right)$ in an airtight plexiglass chamber or normoxia $\left(21 \% \mathrm{O}_{2}\right)$ for 1,2 , or 3 weeks (39). Following 3 weeks of hypoxia, subgroups of animals were reexposed to normoxia for 2,5 , or 9 weeks as described $(43,44)$, for a total of 5,8 , or 12 weeks following SU5416 or vehicle.

\section{Echocardiography}

Five and 8 weeks after SU5416 (3 weeks of hypoxia followed by 2 or 5 weeks of normoxia), rats were anesthetized with isoflurane and echocardiography performed using a Vevo 2100 high-resolution microultrasound system (VisualSonics) and a 24-MHz probe. M-mode and 2-dimensional modalities were used to measure the RV free wall (RVFW) thickness and RV inner diameter (RVID) at end-diastole using the right parasternal long-axis view. Pulsed-wave Doppler was used to measure the pulmonary artery acceleration time (PAAT). To assess RV function, tricuspid annular plane systolic excursion (TAPSE) was recorded in the M-mode from the apical 4-chamber view.

\section{Hemodynamic measurements}

One, 2, 3, and 4 weeks after MCT - and 1, 2, 5, and 8 weeks after SU5416 (3 weeks of hypoxia followed by 2 or 5 weeks of normoxia) — rats were anesthetized with sodium pentobarbital ( $30 \mathrm{mg} / \mathrm{kg}$ ), and hemodynamic measurements were performed as described (39). A 23-g needle was inserted through the diaphragm into the RV, and the RVSP was measured using PowerLab equipment (39). The hearts were excised, ventricles were dissected, and the RV was weighed separately from the left ventricle and septum (LV + S). RVH was assessed by the Fulton's index (RV/LV + S) (39).

\section{Histologic analysis and morphometry}

Lungs were inflated, harvested, fixed in $10 \%$ formalin at $4^{\circ} \mathrm{C}$, and embedded in paraffin. Sections were stained with $\mathrm{H} \& \mathrm{E}(70,71)$. Remodeling was quantitated as described previously (39) by capturing images of H\&E-stained lung tissue ( $5 \mu \mathrm{m}$ sections) with a digital camera (Leica DFC300) coupled to a microscope and measuring arteriolar wall area with ImageJ (NIH). PAs $<100 \mu \mathrm{m}$ and $>100 \mu \mathrm{m}$ in diameter were identified, and at least 10 vessels were evaluated per rat. Percent wall thickness was calculated as: wall thickness $(\%)=\left(\right.$ area $_{\text {ext }}-$ area $\left._{\text {int }}\right) /$ area $_{\text {ext }} \times 100$, where area ext $_{\text {represents the external diameter and area }}$ represents the internal diameter of each vessel (39). The percentage of occluded vessels for each animal was quantitated by capturing images of 4 quadrants (right upper, right lower, left upper, left lower) of H\&E-stained lung tissue ( $\times 100$ magnification) and counting the number of occluded vessels. Vessels occluded with blood were excluded.

\section{Lung parenchymal strip preparation and AFM elasticity measurements}

For rat samples, lungs were inflated with OCT ( $50 \mathrm{ml} / \mathrm{kg}$ body weight), frozen in dry ice-cooled 2-methyl butane, and stored at $-80^{\circ} \mathrm{C}$. Lung parenchymal tissue strips $50 \mu \mathrm{m}$ in thickness were prepared using a cryostat (Leica CM1850), mounted on poly-L-lysine coated glass slides, stored at $-20^{\circ} \mathrm{C}$, and measured within 24 hours. Residual OCT was dissolved and rinsed away with PBS immediately before AFM measurements. Lung parenchymal tissue and PAs $(<100 \mu \mathrm{m},>100 \mu \mathrm{m})$ were mechanically characterized using an MFP- 
3D AFM (Asylum Research) by performing microindentation using a sphere-tipped probe (Novascan) with a diameter of $5 \mu \mathrm{m}$ and a nominal spring constant of approximately $60 \mathrm{pN} / \mathrm{nm}$ as described $(14,38,72)$. The cantilever spring constant was further confirmed by the thermal fluctuation method (73). The AFM system was calibrated by following the manufacturer's instructions before each measurement. Force-indentation profiles were acquired at an indentation rate of $20 \mu \mathrm{m} / \mathrm{s}$, and the shear modulus was calculated by fitting force-indentation data using a Hertz sphere model $(74,75)$. Poisson's ratio $(v)$ of $0.4(76)$ was used to convert elastic modulus (E) to shear modulus $(G)$, using the relationship $E=2 \times(1+v) \times G(14,38)$. For each animal, at least 3 slides per animal were analyzed. For each slide, at least 3 measurements per vessel were made on at least 3 vessels per slide. As lungs were inflated with OCT prior to sample preparation, vessels were identified by morphologic criteria using phase contrast microscopy.

\section{AFM mechanical measurements in human lung tissue}

Human lung tissue was obtained from explanted lungs of patients with Group $1 \mathrm{PAH}$ who underwent lung transplantation or from normal lung tissue obtained from organ donors whose lungs were not suitable for transplantation. Human lung tissues were embedded in OCT and stored at $-80^{\circ} \mathrm{C}$. Lung tissue slices 10 $\mu \mathrm{m}$ in thickness were cut by cryosection at $-23^{\circ} \mathrm{C}$ and mounted on poly-L-lysine-coated glass slides. To avoid drying, PBS was added on the tissue slice. Tissues were incubated with Alexa Fluor 488 anti-human CD31 (BioLegend) diluted at 1:40 in PBS in a humidity chamber for 90 minutes. Vessels were identified by fluorescent microscopy (Olympus) using collagen autofluorescence and CD31 immunofluorescent staining of pulmonary endothelial cells (77).

Human PAs $<100 \mu \mathrm{m}$ and lung parenchymal tissue were mechanically characterized with a BioScope Catalyst AFM (Bruker) by performing microindentation using a sphere-tipped probe (Novascan) with a diameter of $5 \mu \mathrm{m}$ and a spring constant of approximately $98 \mathrm{pN} / \mathrm{nm}$ determined by the thermal fluctuation method (73). For each vessel, 5 force curves on different areas along the vessel were acquired with MIRO 2.0 (NanoScope 9.1, Bruker) at room temperature in PBS at an indentation rate of $20 \mu \mathrm{m} / \mathrm{s}$ and a ramp size of $10 \mu \mathrm{m}$. The indentation was estimated at approximately $250 \mathrm{~nm}$ for an applied force of approximately 26 $\mathrm{nN}$. Force curves were analyzed by NanoScope Analysis (Bruker), and the shear modulus was determined by fitting force-indentation data using a Hertz sphere model $(74,75)$. Poisson's ratio of 0.4 (76) was used to convert elastic modulus $(\mathrm{E})$ to shear modulus $(\mathrm{G})$, using the relationship $\mathrm{E}=2 \times(1+v) \times \mathrm{G}(14,38)$.

\section{Metabololipidomics}

Lipid mediator metabololipidomics was performed as described (78). Briefly, lung tissues were weighed and gently dispersed in ice-cold methanol containing an internal standard $\left(\mathrm{d}_{4}-\mathrm{PGE}_{2}, 500 \mathrm{pg}\right)$ to facilitate quantification and recovery. Samples were held at $-20^{\circ} \mathrm{C}$ for 45 minutes to allow protein precipitation and were centrifuged. Supernatants were collected and placed onto an automated extraction system (Biotage) using a C18 column. Prostanoids were collected and injected onto an LC system (Shimadzu) coupled to a Qtrap 5500 (ABSciex). Identification was conducted using published criteria including matching retention time to synthetic standards and diagnostic ions in the tandem mass spectometry (MS-MS) mode.

\section{Cell culture}

Human PASMC (hPASMC) were purchased from Lonza and cultured in smooth muscle basal medium (SmBM) supplemented with 5\% FBS, SmGM-2 SingleQuots (Lonza), penicillin (100 IU/m), and streptomycin $(100 \mu \mathrm{g} / \mathrm{ml})$ in a humidified incubator $\left(21 \% \mathrm{O}_{2}, 5 \% \mathrm{CO}_{2}\right)$ at $37^{\circ} \mathrm{C}$. Human PAEC (hPAEC, Lonza) were cultured in endothelial cell basal medium-2 (EBM-2) supplemented with 2\% FBS, EGM-2 SingleQuots (Lonza), penicillin (100 IU/ml), and streptomycin $(100 \mu \mathrm{g} / \mathrm{ml})$ in a humidified incubator $\left(21 \% \mathrm{O}_{2}\right.$, $5 \% \mathrm{CO}_{2}$ ) at $37^{\circ} \mathrm{C}$. Experiments were performed on hPASMC at passages $7-10$ and hPAEC at passages 6-8. Primary PASMC were isolated from adult (8-10 weeks old) COX-2 $2^{-/}$and COX-2 ${ }^{+/+}$mice as described (39) and cultured in DMEM containing 20\% FBS; 10,000 IU/ml penicillin; 10,000 $\mu \mathrm{g} / \mathrm{ml}$ streptomycin; 29.2 $\mathrm{mg} / \mathrm{ml} \mathrm{L}$-glutamine; $1 \mathrm{mM}$ sodium pyruvate; and $1 \times \mathrm{MEM}$ nonessential amino acids in a humidified incubator $\left(21 \% \mathrm{O}_{2}, 5 \% \mathrm{CO}_{2}\right)$ at $37^{\circ} \mathrm{C}$. Experiments were performed on PASMC at passages 6-10.

Normal and BMPR2-mutant PASMC were derived at the Cleveland Clinic Foundation from a control subject and a subject with BMPR2 mutation, respectively (79). PASMC were isolated from elastic pulmonary arteries ( $>500 \mu \mathrm{m}$ diameter) dissected from explanted lungs by collagenase/DNase digestion and characterized by IHC as described (79). Cells were cultured in 15 mmol/1 HEPES-buffered DMEM/F12 
(Invitrogen) supplemented with 10\% FBS (Hyclone) and 2.5\% penicillin/streptomycin/amphotericin B (Invitrogen) in a humidified incubator $\left(21 \% \mathrm{O}_{2}, 5 \% \mathrm{CO}_{2}\right)$ at $37^{\circ} \mathrm{C}$. Primary cultures of passages $7-10$ were used in experiments.

\section{Discrete stiffness gel preparation}

Gels were prepared to fit 24-well plates with individual discrete stiffness (shear modulus) of $0.1,0.4,1.6$, 6.4 , and $25.6 \mathrm{kPa}$ as described with minor modifications $(14,45)$. Briefly, 15-mm glass coverslips (Fisher Scientific) were silanized with $0.4 \%$ 3-(Trimethoxysilyl)propyl methacrylate (Sigma-Aldrich) solution in acetone. A glass plate of 3-by-4 inches (Bio-Rad) was treated with SurfaSil (ThermoFisher) to generate a hydrophobic surface. Solutions $(20 \mu \mathrm{l})$ containing $0.075 \%$ ammonium persulfate, $0.15 \%$ tetramethylethylenediamine, and variable ratios of acrylamide/bisacrylamide (Bio-Rad) were delivered onto the hydrophobic glass plate surface and sandwiched with silanized coverslips. Following polymerization, the gels were microwave-sterilized and placed into a 24-well plate. The gel surface was derivatized with the heterobifunctional crosslinker sulfo-SANPAH (ThermoFisher) as previously described (28). Monomeric collagen (PureCol, Advanced Biomatrix) diluted in PBS at $10 \mathrm{mg} / \mathrm{ml}$ was delivered to each well and incubated for 4 hours at room temperature. The plate was rinsed in PBS prior to cell seeding.

\section{Lentiviral transduction}

COX-2 was overexpressed in hPASMC using the pLOC lentiviral expression vector containing the ORF for human PTGS2 with the native stop codon (Precision LentiORF Collection, Thermo Scientific). PASMC were plated at 3,000 cells/well in a 96-well plate and, the following day, were transduced with pLOC-COX-2 or pLOC-RFP control viral particles at an optimized MOI of 50 in serum- and antibiotic-free SmBM containing $1 \times$ polybrene. After 2 days, the cells were selected with blasticidin $(5 \mu \mathrm{g} / \mathrm{ml})$ for 5 days. COX-2 expression in transduced PASMC was determined by qPCR and Western blot analysis. Stable COX-2expressing PASMC were grown in SmBM with 5\% FBS, SmGM-2 SingleQuots, penicillin (100 IU/ml), and streptomycin $(100 \mu \mathrm{g} / \mathrm{ml})$, along with continuous exposure to the selection reagent blasticidin $(1 \mu \mathrm{g} / \mathrm{ml})$.

\section{Proliferation and apoptosis assays}

hPASMC and hPAEC were seeded at a density of $25 \mathrm{cell} / \mathrm{mm}^{2}$ and $50 \mathrm{cell} / \mathrm{mm}^{2}$, respectively, in 24-well plates containing gels with discrete stiffness of $0.1,0.4,1.6,6.4$, and $25.6 \mathrm{kPa}$ and cultured at $37^{\circ} \mathrm{C}$ and $5 \% \mathrm{CO}_{2}$. For hypoxia experiments, hPASMC were placed in an airtight Modular Incubator Chamber (Billups-Rothenberg), flushed continuously for 20 minutes with a premixed gas of $1 \% \mathrm{O}_{2}, 5 \% \mathrm{CO}_{2}, 94 \% \mathrm{~N}_{2}$ (hypoxia) or $95 \%$ air with $5 \% \mathrm{CO}_{2}$ (normoxia) and incubated at $37^{\circ} \mathrm{C}$ for 48 hours. In specific experiments, hPASMC were treated with iloprost ( 3 and $10 \mu \mathrm{mol} / 1$ ) or vehicle (DMSO) 4 hours following plating and at 24 hours. After 48 hours, cell density was determined using the CyQuant NF Cell Proliferation Assay (Invitrogen). For each stiffness condition, fold change was expressed as the ratio of adherent cell number at 48 versus 4 hours. In separate experiments, hPASMC and hPAEC were plated on discrete stiffness gels, and after 48 hours, apoptosis was assessed using the Apo-ONE Homogeneous Caspase-3/ 7 Assay (Promega). Percent apoptosis was normalized to maximum apoptosis at $0.1 \mathrm{kPa}$.

\section{Immunofluorescent staining}

hPASMC were seeded on 24-well plates containing gels with stiffness of 0.4 and $25.6 \mathrm{kPa}$ and treated with iloprost $(10 \mu \mathrm{mol} / 1)$ or vehicle (DMSO). After 48 hours, cells were fixed with $4 \%$ formalin, blocked with $5 \%$ goat serum, and stained with mouse monoclonal anti-Pro-Collagen I (clone PCIDG10, catalog MAB1913, Millipore) and mouse monoclonal anti-Fibronectin (clone IST-9, Abcam) antibodies. After washing, cells were incubated with Alexa Fluor 546 goat anti-mouse IgG1 (Invitrogen). F-actin and nuclei were stained with Alexa Fluor 488-phalloidin and Hoechst 33342 (Invitrogen), respectively. Fluorescent images were obtained with a Nikon TE300 fluorescent microscope. The final image contrast was adjusted with MetaMorph 6.1 (Universal Imaging) by setting the background to the fluorescence intensity level of the samples in the corresponding secondary antibody-only control images. Procollagen I staining was quantified with MetaMorph 6.1 by counting the procollagen I staining-positive cells and normalizing to the total cell number in each image. Fibronectin staining was quantified with MetaMorph 6.1 by measuring the average cellular fibronectin fluorescence intensity (normalized to stained areas) and then normalized to the corresponding total cell number. 


\section{PCR array and qPCR}

hPASMC were seeded at a density of $50 \mathrm{cell} / \mathrm{mm}^{2}$ and $\mathrm{hPAEC}$ at $100 \mathrm{cell} / \mathrm{mm}^{2}$ in 24 -well plates containing gels with stiffness of $0.1,0.4,6.4$, and $25.6 \mathrm{kPa}$ and cultured at $37^{\circ} \mathrm{C}$ and $5 \% \mathrm{CO}_{2}$. After 48 hours, cells in 8-12 replicate stiffness wells were consecutively lysed with $350 \mu 1$ RNA lysis buffer (Qiagen), and RNA was isolated using the RNeasy Mini Kit (Qiagen). RNA was treated with DNase (Invitrogen) followed by reverse transcription to cDNA using the Superscript III qRT-PCR Kit (Invitrogen). Equivalent amounts of cDNA were added to RT ${ }^{2}$ SYBR Green qPCR Mastermix (Qiagen) containing $1 \mu \mathrm{mol} / 1$ each of forward and reverse primers, and qPCR was performed on a 7300 Real-Time PCR System (Applied Biosystems) to determine gene expression for PTGS1 (COX-1), PTGS2 (COX-2), PGI2R (PGI r receptor), COL1A1 (type 1 collagen), and FN1 (fibronectin 1). Primers were designed with qPrimerDepot (http://primerdepot.nci. nih.gov) and are intron overlapping (Supplemental Table 4). All qPCR results were normalized to the expression of the endogenous positive control GAPDH. The $\triangle \mathrm{Ct}$ was calculated as the difference between the $\mathrm{Ct}$ for the gene of interest and the respective $\mathrm{Ct}$ for GAPDH. For the PCR Array, RNA was converted to cDNA using the RT ${ }^{2}$ First Strand Kit and added to the RT ${ }^{2}$ SYBR Green Mastermix, and qPCR was performed using the Human Hypertension RT ${ }^{2}$ Profiler PCR Array (all from Qiagen).

For qPCR in rat tissue, gene expression for COL1A1 (Rn01463848_m1), COL3A1 (Rn01437681_m1), FN1 (Rn00569575_m1), and LOX (lysyl oxidase; Rn01491829_m1) was determined using validated TaqMan gene expression assay primer/probe combinations (ThermoFisher). All qPCR results were normalized to the expression of the endogenous positive control 18S (Hs99999901_s1).

\section{Transient transfection}

A $1-\mathrm{kb}$ fragment of the murine COX-2 5'-flanking sequence $(-976 /+56)$ was subcloned into the PGL2-basic luciferase reporter plasmid ( $\left.5^{\prime} \mathrm{Xho} \mathrm{I} / 3^{\prime} \mathrm{Hind} \mathrm{III}\right)$. hPASMC were transiently transfected with the mCOX-2 promoter luciferase construct and pCMV- $\beta$-gal via electroporation (400 V, $10 \mathrm{msec}$, single pulse) using the Gene Pulser Xcell Electroporation System (Bio-Rad). Transfected cells were seeded on synthetic polyacrylamide substrates of defined stiffness spanning a shear modulus range of $0.1-25.6 \mathrm{kPa}$ in a 24 -well plate. After 24 hours, cells were harvested and luciferase and $\beta$-galactosidase assays performed.

\section{ELISA}

hPASMC and hPAEC were seeded at a density of $25 \mathrm{cell} / \mathrm{mm}^{2}$ and $50 \mathrm{cell} / \mathrm{mm}^{2}$, respectively, in 24-well plates containing gels with stiffness of $0.1,0.4,1.6,6.4$, and $25.6 \mathrm{kPa}$ and cultured at $37^{\circ} \mathrm{C}$ and $5 \% \mathrm{CO}_{2}$. After 48 hours, cell culture media were collected and analyzed for $\mathrm{PGE}_{2}$ and 6-keto-PGF ${ }_{1 \alpha}$ concentrations by ELI$\mathrm{SA}$ (Cayman). $\mathrm{PGE}_{2}$ and 6-keto-PGF ${ }_{1 \alpha}$ concentrations were normalized to the corresponding cell numbers (pg/cell) as measured by CyQuant Assay.

\section{Soluble collagen assay}

Human PASMC were seeded at a density of $50 \mathrm{cell} / \mathrm{mm}^{2}$ in 24 -well plates containing gels with stiffness of 0.4 and $25.6 \mathrm{kPa}$. Cells were cultured with standard media containing $5 \% \mathrm{FBS}$ at $37^{\circ} \mathrm{C}$ and $5 \% \mathrm{CO}_{2}$. After 48 hours, cell culture media were collected, and the collagen concentration was measured using a Sircol Soluble Collagen Assay Kit according to manufacturer's instructions (Biocolor). Collagen concentrations were normalized to the corresponding cell numbers (pg/cell) as measured by CyQuant Assay.

\section{Traction force microscopy}

Contractile forces exerted by PASMC on different stiffness gels were assessed by traction force microscopy essentially as described $(39,46,47)$. Briefly, polyacrylamide substrates with shear moduli of $0.4,1.6$, and $6.4 \mathrm{kPa}$ were prepared $(14,45)$ and fluorescent sulfate-modified latex microspheres $(0.2$ $\mu \mathrm{m}, 505 / 515 \mathrm{ex} / \mathrm{em}$, FluoSpheres, Invitrogen) were conjugated to the gel surfaces as described (47). PASMC were plated on fluorescent bead-conjugated discrete stiffness gels and grown for 24 hours, at which time they were treated with iloprost $(10 \mu \mathrm{mol} / 1)$ or vehicle for 30 minutes before traction force measurements. Images of gel surface-conjugated fluorescent beads were acquired for each cell before and after trypsinization using a Pathway HT fluorescence imaging system (BD Biosciences) and a $\times 20$ magnification objective. Cell area was visualized using CellLight Plasma Membrane-RFP (Invitrogen) as described (47) to delineate the cell perimeter. Tractions exerted by PASMC were estimated by measuring bead displacement fields, computing corresponding traction fields using Fourier transform 
traction microscopy (46), and calculating root-mean-square traction (RMST) with MATLAB (MathWorks) (46).

\section{Statistics}

Data are presented as mean \pm SEM unless otherwise specified. Statistical significance was determined by the nonparametric Mann-Whitney $U$ test or unpaired $t$ test for comparisons between 2 groups; the nonparametric Kruskal-Wallis 1-way ANOVA was used for comparisons between more than 2 groups. Two-way ANOVA was used to determine significance of a response to 2 or more factors. Multivariable data were fitted to a mixed model (JMP version 12.0, SAS Institute) using fixed, nested, and random effects as appropriate to account for multiple AFM measurements obtained from the same animal or human subject at a given time point; multiple comparisons were performed using Dunnett's test. Data plotted according to the individual animal or human subject are presented in the Supplemental Data. The number of samples per group $(n)$ is specified in the figure legends. All $P$ values were 2-tailed, and statistical significance was accepted at $P<0.05$.

\section{Study approval}

All animal experiments were performed in compliance with the relevant laws and guidelines as set forth by the Harvard Medical Area Standing Committee on Animals and the Lovelace Respiratory Research Institute Animal Care and Use Committee. Human tissue samples and cell lines were obtained from the Pulmonary Hypertension Breakthrough Initiative (PHBI) and Cleveland Clinic, respectively, under a protocol approved by the Partners Human Research Committee. Informed consent was obtained by the PHBI from the subjects or their legal guardians prior to enrolling in the study.

\section{Author contributions}

LEF and DJT designed the studies and wrote the manuscript. FL, CMH, PBD, DS, IC, AMFC, MMSV, SV, RAC, PCN, AM, XL, JM, CDR, SJL, JDM, CNS, and SRW performed experiments, acquired and/or analyzed data, and assisted in manuscript preparation. SAAC and SCE provided human cell lines.

\section{Acknowledgments}

This work was supported by an American Thoracic Society/Pulmonary Hypertension Association/Pfizer Research Fellowship in Pulmonary Arterial Hypertension (to L.E. Fredenburgh), as well as grants from the NIH including K08 HL077344 (to S. Vitali), T32 HL007633 (to P.B. Dieffenbach), T32 HL007118 (to A. Marinković), R37 HL060917 (to S.C. Erzurum), P01 HL103453 (to S.C. Erzurum), R01 HL092961 (to D.J. Tschumperlin), R01 HL114839 (to L.E. Fredenburgh and D.J. Tschumperlin), R03 HL115106 (to L.E. Fredenburgh), P01 HL108801 (to L.E. Fredenburgh and C.N. Serhan), and K08 GM083207 (to L.E. Fredenburgh). Funding for the PHBI is provided by the Cardiovascular Medical Research and Education Fund (CMREF). This work was performed in part at the Center for Nanoscale Systems (CNS), a member of the National Nanotechnology Infrastructure Network (NNIN), which is supported by the National Science Foundation under NSF award ECS-0335765. This work was performed in part at the Center for Nanoscale Systems, a member of the National Nanotechnology Coordinated Infrastructure Network (NNCI), which is supported by the National Science Foundation under NSF award no. 1541959. Center for Nanoscale Systems is part of Harvard University. The authors thank Emeka Ifedigbo for technical assistance and Lester Kobzik for use of the cryosection, as well as Matthew D. Layne, Bruce D. Levy, and Shelley Hurwitz for helpful discussions.

Address correspondence to: Laura E. Fredenburgh, Division of Pulmonary and Critical Care Medicine, Brigham and Women's Hospital, 75 Francis Street, Boston, Massachusetts 02115, USA. Phone: 617.525.9563; E-mail: 1fredenburgh@rics.bwh.harvard.edu.

\footnotetext{
1. Fredenburgh LE, Ma J, Perrella MA. Cyclooxygenase-2 inhibition and hypoxia-induced pulmonary hypertension: effects on pulmonary vascular remodeling and contractility. Trends Cardiovasc Med. 2009;19(2):31-37.

2. Kaess BM, et al. Aortic stiffness, blood pressure progression, and incident hypertension. JAMA. 2012;308(9):875-881.

3. Laurent $\mathrm{S}$, et al. Aortic stiffness is an independent predictor of all-cause and cardiovascular mortality in hypertensive patients. Hypertension. 2001;37(5):1236-1241.

4. Blacher J, Guerin AP, Pannier B, Marchais SJ, Safar ME, London GM. Impact of aortic stiffness on survival in end-stage renal
} 
disease. Circulation. 1999;99(18):2434-2439.

5. Gan CT, et al. Noninvasively assessed pulmonary artery stiffness predicts mortality in pulmonary arterial hypertension. Chest. 2007;132(6):1906-1912.

6. Rodes-Cabau J, et al. Intravascular ultrasound of the elastic pulmonary arteries: a new approach for the evaluation of primary pulmonary hypertension. Heart. 2003;89(3):311-315.

7. Mahapatra S, Nishimura RA, Sorajja P, Cha S, McGoon MD. Relationship of pulmonary arterial capacitance and mortality in idiopathic pulmonary arterial hypertension. J Am Coll Cardiol. 2006;47(4):799-803.

8. Hunter KS, et al. Pulmonary vascular input impedance is a combined measure of pulmonary vascular resistance and stiffness and predicts clinical outcomes better than pulmonary vascular resistance alone in pediatric patients with pulmonary hypertension. Am Heart J. 2008;155(1):166-174.

9. Wang Z, Chesler NC. Pulmonary vascular wall stiffness: An important contributor to the increased right ventricular afterload with pulmonary hypertension. Pulm Circ. 2011;1(2):212-223.

10. Stevens GR, Garcia-Alvarez A, Sahni S, Garcia MJ, Fuster V, Sanz J. RV dysfunction in pulmonary hypertension is independently related to pulmonary artery stiffness. JACC Cardiovasc Imaging. 2012;5(4):378-387.

11. Kang KW, et al. Cardiac magnetic resonance imaging-derived pulmonary artery distensibility index correlates with pulmonary artery stiffness and predicts functional capacity in patients with pulmonary arterial hypertension. Circ J. 2011;75(9):2244-2251.

12. Klein EA, et al. Cell-cycle control by physiological matrix elasticity and in vivo tissue stiffening. Curr Biol. 2009;19(18):15111518 .

13. Liu F, et al. Mechanosignaling through YAP and TAZ drives fibroblast activation and fibrosis. Am J Physiol Lung Cell Mol Physiol. 2015;308(4):L344-L357.

14. Liu F, et al. Feedback amplification of fibrosis through matrix stiffening and COX-2 suppression. J Cell Biol. 2010;190(4):693706.

15. Ooi CY, Wang Z, Tabima DM, Eickhoff JC, Chesler NC. The role of collagen in extralobar pulmonary artery stiffening in response to hypoxia-induced pulmonary hypertension. Am J Physiol Heart Circ Physiol. 2010;299(6):H1823-H1831.

16. Kobs RW, Muvarak NE, Eickhoff JC, Chesler NC. Linked mechanical and biological aspects of remodeling in mouse pulmonary arteries with hypoxia-induced hypertension. Am J Physiol Heart Circ Physiol. 2005;288(3):H1209-H1217.

17. Tabima DM, Chesler NC. The effects of vasoactivity and hypoxic pulmonary hypertension on extralobar pulmonary artery biomechanics. J Biomech. 2010;43(10):1864-1869.

18. Tuchscherer HA, Vanderpool RR, Chesler NC. Pulmonary vascular remodeling in isolated mouse lungs: effects on pulsatile pressure-flow relationships. J Biomech. 2007;40(5):993-1001.

19. Wang Z, Chesler NC. Role of collagen content and cross-linking in large pulmonary arterial stiffening after chronic hypoxia. Biomech Model Mechanobiol. 2011;11(1-2):279-289.

20. Drexler ES, et al. Stiffening of the extrapulmonary arteries from rats in chronic hypoxic pulmonary hypertension. $J$ Res Natl Stand Technol. 2008;113(4):239-249.

21. Hunter KS, et al. In vivo measurement of proximal pulmonary artery elastic modulus in the neonatal calf model of pulmonary hypertension: development and ex vivo validation. J Appl Physiol. 2010;108(4):968-975.

22. Lammers SR, et al. Changes in the structure-function relationship of elastin and its impact on the proximal pulmonary arterial mechanics of hypertensive calves. Am J Physiol Heart Circ Physiol. 2008;295(4):H1451-H1459.

23. Nave AH, et al. Lysyl oxidases play a causal role in vascular remodeling in clinical and experimental pulmonary arterial hypertension. Arterioscler Thromb Vasc Biol. 2014;34(7):1446-1458.

24. Schafer M, et al. Pulmonary arterial stiffness: Toward a new paradigm in pulmonary arterial hypertension pathophysiology and assessment. Curr Hypertens Rep. 2016;18(1):4.

25. Tschumperlin DJ. Matrix, mesenchyme, and mechanotransduction. Ann Am Thorac Soc. 2015;12(supp1 1):S24-S29.

26. Discher DE, Janmey P, Wang YL. Tissue cells feel and respond to the stiffness of their substrate. Science. 2005;310(5751):11391143.

27. Mammoto T, Ingber DE. Mechanical control of tissue and organ development. Development. 2010;137(9):1407-1420.

28. Pelham RJ Jr, Wang Y. Cell locomotion and focal adhesions are regulated by substrate flexibility. Proc Natl Acad Sci USA. 1997;94(25):13661-13665.

29. Engler AJ, Sen S, Sweeney HL, Discher DE. Matrix elasticity directs stem cell lineage specification. Cell. 2006;126(4):677-689.

30. Ivanovska IL, Shin JW, Swift J, Discher DE. Stem cell mechanobiology: diverse lessons from bone marrow. Trends Cell Biol. 2015;25(9):523-532.

31. Shin JW, et al. Contractile forces sustain and polarize hematopoiesis from stem and progenitor cells. Cell Stem Cell. 2014;14(1):81-93.

32. Caiazzo M, Okawa Y, Ranga A, Piersigilli A, Tabata Y, Lutolf MP. Defined three-dimensional microenvironments boost induction of pluripotency. Nat Mater. 2016;15(3):344-352.

33. Paszek MJ, et al. Tensional homeostasis and the malignant phenotype. Cancer Cell. 2005;8(3):241-254.

34. Taubenberger AV, et al. 3D extracellular matrix interactions modulate tumour cell growth, invasion angiogenesis in engineered tumour microenvironments. Acta Biomater. 2016;36:73-85.

35. Peyton SR, Putnam AJ. Extracellular matrix rigidity governs smooth muscle cell motility in a biphasic fashion. J Cell Physiol. 2005;204(1):198-209.

36. Engler A, Bacakova L, Newman C, Hategan A, Griffin M, Discher D. Substrate compliance versus ligand density in cell on gel responses. Biophys J. 2004;86(1 pt 1):617-628.

37. Shkumatov A, et al. Matrix stiffness-modulated proliferation and secretory function of the airway smooth muscle cells. Am $J$ Physiol Lung Cell Mol Physiol. 2015;308(11):L1125-L1135.

38. Liu F, Tschumperlin DJ. Micro-mechanical characterization of lung tissue using atomic force microscopy. $J$ Vis Exp. 2011;(54):2911.

39. Fredenburgh LE, et al. Absence of cyclooxygenase-2 exacerbates hypoxia-induced pulmonary hypertension and enhances contractility of vascular smooth muscle cells. Circulation. 2008;117(16):2114-2122. 
40. Tuder RM, et al. Prostacyclin synthase expression is decreased in lungs from patients with severe pulmonary hypertension. Am $J$ Respir Crit Care Med. 1999;159(6):1925-1932.

41. Christman BW, et al. An imbalance between the excretion of thromboxane and prostacyclin metabolites in pulmonary hypertension. N Engl J Med. 1992;327(2):70-75.

42. Falcetti E, et al. Smooth muscle proliferation and role of the prostacyclin (IP) receptor in idiopathic pulmonary arterial hypertension. Am J Respir Crit Care Med. 2010;182(9):1161-1170.

43. Abe K, et al. Formation of plexiform lesions in experimental severe pulmonary arterial hypertension. Circulation. 2010;121(25):2747-2754.

44. Taraseviciene-Stewart L, et al. Inhibition of the VEGF receptor 2 combined with chronic hypoxia causes cell death-dependent pulmonary endothelial cell proliferation and severe pulmonary hypertension. FASEB J. 2001;15(2):427-438

45. Mih JD, Sharif AS, Liu F, Marinkovic A, Symer MM, Tschumperlin DJ. A multiwell platform for studying stiffness-dependent cell biology. PLoS One. 2011;6(5):e19929.

46. Butler JP, Tolic-Norrelykke IM, Fabry B, Fredberg JJ. Traction fields, moments, and strain energy that cells exert on their surroundings. Am J Physiol Cell Physiol. 2002;282(3):C595-C605.

47. Marinkovic A, Mih JD, Park JA, Liu F, Tschumperlin DJ. Improved throughput traction microscopy reveals pivotal role for matrix stiffness in fibroblast contractility and TGF-beta responsiveness. Am J Physiol Lung Cell Mol Physiol. 2012;303(3):L169L180.

48. Lau EM, Iyer N, Ilsar R, Bailey BP, Adams MR, Celermajer DS. Abnormal pulmonary artery stiffness in pulmonary arterial hypertension: in vivo study with intravascular ultrasound. PLoS One. 2012;7(3):e33331.

49. Sanz J, et al. Evaluation of pulmonary artery stiffness in pulmonary hypertension with cardiac magnetic resonance. JACC Cardiovasc Imaging. 2009;2(3):286-295.

50. Mahapatra S, Nishimura RA, Oh JK, McGoon MD. The prognostic value of pulmonary vascular capacitance determined by Doppler echocardiography in patients with pulmonary arterial hypertension. J Am Soc Echocardiogr. 2006;19(8):1045-1050.

51. Weinberg CE, et al. Extraction of pulmonary vascular compliance, pulmonary vascular resistance, and right ventricular work from single-pressure and Doppler flow measurements in children with pulmonary hypertension: a new method for evaluating reactivity: in vitro and clinical studies. Circulation. 2004;110(17):2609-2617.

52. Kobs RW, Chesler NC. The mechanobiology of pulmonary vascular remodeling in the congenital absence of eNOS. Biomech Model Mechanobiol. 2006;5(4):217-225.

53. Tan W, Madhavan K, Hunter KS, Park D, Stenmark KR. Vascular stiffening in pulmonary hypertension: cause or consequence? (2013 Grover Conference series). Pulm Circ. 2014;4(4):560-580.

54. Benyahia C, et al. Ex vivo relaxations of pulmonary arteries induced by prostacyclin mimetics are highly dependent of the precontractile agents. Prostaglandins Other Lipid Mediat. 2015;121(pt A):46-52.

55. Clapp LH, Gurung R. The mechanistic basis of prostacyclin and its stable analogues in pulmonary arterial hypertension: Role of membrane versus nuclear receptors. Prostaglandins Other Lipid Mediat. 2015;120:56-71.

56. Orie NN, Ledwozyw A, Williams DJ, Whittle BJ, Clapp LH. Differential actions of the prostacyclin analogues treprostinil and iloprost and the selexipag metabolite, MRE-269 (ACT-333679) in rat small pulmonary arteries and veins. Prostaglandins Other Lipid Mediat. 2013;106:1-7.

57. Whittle BJ, Silverstein AM, Mottola DM, Clapp LH. Binding and activity of the prostacyclin receptor (IP) agonists, treprostinil and iloprost, at human prostanoid receptors: treprostinil is a potent DP1 and EP2 agonist. Biochem Pharmacol. 2012;84(1):68-75

58. Lang IM, Gaine SP. Recent advances in targeting the prostacyclin pathway in pulmonary arterial hypertension. Eur Respir Rev. 2015;24(138):630-641.

59. McLaughlin VV, et al. ACCF/AHA 2009 expert consensus document on pulmonary hypertension a report of the American College of Cardiology Foundation Task Force on Expert Consensus Documents and the American Heart Association developed in collaboration with the American College of Chest Physicians; American Thoracic Society, Inc.; and the Pulmonary Hypertension Association. J Am Coll Cardiol. 2009;53(17):1573-1619.

60. Farber HW, Miller DP, Meltzer LA, McGoon MD. Treatment of patients with pulmonary arterial hypertension at the time of death or deterioration to functional class IV: insights from the REVEAL Registry. J Heart Lung Transplant. 2013;32(11):11141122 .

61. Delcroix M, Spaas K, Quarck R. Long-term outcome in pulmonary arterial hypertension: a plea for earlier parenteral prostacyclin therapy. Eur Respir Rev. 2009;18(114):253-259.

62. Vachiery JL. Prostacyclins in pulmonary arterial hypertension: the need for earlier therapy. Adv Ther. 2011;28(4):251-269.

63. Jing ZC, et al. Efficacy and safety of oral treprostinil monotherapy for the treatment of pulmonary arterial hypertension: a randomized, controlled trial. Circulation. 2013;127(5):624-633.

64. Simonneau G, et al. Selexipag: an oral, selective prostacyclin receptor agonist for the treatment of pulmonary arterial hypertension. Eur Respir J. 2012;40(4):874-880.

65. Tschumperlin DJ, Boudreault F, Liu F. Recent advances and new opportunities in lung mechanobiology. J Biomech. 2010;43(1):99-107.

66. Huynh J, et al. Age-related intimal stiffening enhances endothelial permeability and leukocyte transmigration. Sci Transl Med. 2011;3(112):112ra122.

67. Hayenga HN, Trache A, Trzeciakowski J, Humphrey JD. Regional atherosclerotic plaque properties in ApoE $E^{-/-}$mice quantified by atomic force, immunofluorescence, and light microscopy. J Vasc Res. 2011;48(6):495-504.

68. Liu SL, et al. Matrix metalloproteinase-12 is an essential mediator of acute and chronic arterial stiffening. Sci Rep 2015;5:17189.

69. Lalich JJ, Merkow L. Pulmonary arteritis produced in rat by feeding Crotalaria spectabilis. Lab Invest. 1961;10:744-750.

70. Fredenburgh LE, et al. Absence of heme oxygenase-1 expression in the lung parenchyma exacerbates endotoxin-induced acute lung injury and decreases surfactant protein-B levels. Cell Mol Biol. 2005;51(5):513-520

71. Fredenburgh LE, et al. Cyclooxygenase-2 deficiency leads to intestinal barrier dysfunction and increased mortality during polymicrobial sepsis. J Immunol. 2011;187(10):5255-5267. 
72. Samuel MS, et al. Actomyosin-mediated cellular tension drives increased tissue stiffness and $\beta$-catenin activation to induce epidermal hyperplasia and tumor growth. Cancer Cell. 2011;19(6):776-791.

73. Thundat T, Warmack RJ, Chen GY, Allison DP. Thermal and ambient-induced deflections of scanning force microscope cantilevers. Appl Phys Lett. 1994;64:2894-2896.

74. Dimitriadis EK, Horkay F, Maresca J, Kachar B, Chadwick RS. Determination of elastic moduli of thin layers of soft material using the atomic force microscope. Biophys J. 2002;82(5):2798-2810.

75. Hertz H. Über die Berührung fester elastischer Körper (On the contact of elastic solids). J Reine Angew Mathematik. $1881 ; 92: 156-171$.

76. Butler JP, Nakamura M, Sasaki H, Sasaki T, Takishima T. Poissons' ratio of lung parenchyma and parenchymal interaction with bronchi. Jpn J Physiol. 1986;36(1):91-106.

77. Pusztaszeri MP, Seelentag W, Bosman FT. Immunohistochemical expression of endothelial markers CD31, CD34, von Willebrand factor, and Fli-1 in normal human tissues. J Histochem Cytochem. 2006;54(4):385-395.

78. Colas RA, Shinohara M, Dalli J, Chiang N, Serhan CN. Identification and signature profiles for pro-resolving and inflammatory lipid mediators in human tissue. Am J Physiol Cell Physiol. 2014;307(1):C39-C54.

79. Comhair SA, Xu W, Mavrakis L, Aldred MA, Asosingh K, Erzurum SC. Human primary lung endothelial cells in culture. Am J Respir Cell Mol Biol. 2012;46(6):723-730. 